Bull. Soc. math. France

130 (1), 2002, p. $123-168$

\title{
MESURES SEMI-CLASSIQUES ET CROISEMENT DE MODES
}

\author{
Par Clotilde Fermanian-Kammerer \& Patrick GÉrard
}

\begin{abstract}
RÉsumé. - L'étude de la dynamique semi-classique d'électrons dans un cristal débouche naturellement sur le problème de l'évolution des mesures semi-classiques en présence d'un croisement de modes. Dans ce travail, nous étudions un système $2 \times 2$ qui présente un tel croisement. À cet effet, nous introduisons des mesures semiclassiques à deux échelles qui décrivent comment la transformée de Wigner usuelle se concentre sur l'ensemble des trajectoires rencontrant ce croisement. Puis nous établissons des formules explicites de type Landau-Zener reliant les traces de ces mesures de part et d'autre du croisement.

AbStract (Semi-classical measures and eigenvalue crossings). - Semiclassical study of multidimensional crystals leads naturally to the following question: how do semiclassical measures propagate through energy level crossings ? In this contribution, we discuss a simple $2 \times 2$ system which displays such a crossing. For that purpose, we introduce two-scaled semi-classical measures, which describe how the usual Wigner transforms are concentrating on trajectories passing through the crossing points. Then we derive explicit formulae for the branching of such measures. These formulae are generalizations of the so-called Landau-Zener formulae.
\end{abstract}

Texte reçu le 6 avril 2001, accepté le 3 juillet 2001

Clotilde Fermanian-Kammerer, Université de Cergy-Pontoise, Mathématiques, 2 avenue Adolphe Chauvin, BP 222, 95302 Cergy-Pontoise Cedex (France)

E-mail : Clotilde.Fermanian@math.u-cergy.fr

PATRICK GÉRARd, Université Paris XI, Mathématiques, Bât. 425, 91405 Orsay (France)

E-mail : Patrick.GERARD@math.u-psud.fr

Classification mathématique par sujets (2000). — 35B27, 35L, 35Q40.

Mots clefs. - Mesures semi-classiques (de Wigner), valeurs propres de multiplicité variable, formule de Landau-Zener, convergence à deux échelles.

Le présent travail a été réalisé avec le support du réseau européen TMR « Asymptotic Methods in Kinetic Equations », contrat ERB FMRX CT97 057.

BULletin DE LA SOCiÉtÉ MATHÉMATiQUE DE FRANCE

$0037-9484 / 2002 / 123 / \$ 5.00$ 


\section{Introduction}

1.1. Position du problème. — La dynamique semi-classique d'électrons dans un cristal a fait l'objet de plusieurs travaux récents (voir par exemple [8], [11], [22], [26]). Dans le cas d'un cristal multidimensionnel, l'analyse de ce problème se heurte au fait désormais bien connu que le hamiltonien classique possède des valeurs propres de multiplicité variable (voir par exemple [4]), de sorte que les modes peuvent éventuellement interagir. C'est ce type de difficulté que nous nous proposons de résoudre ici dans un cas simple.

Fixons d'abord les notations. Pour $\xi=\left(\xi_{1}, \xi_{2}\right) \in \mathbb{R}^{2}$, la matrice

$$
A(\xi)=\left(\begin{array}{cc}
\xi_{1} & \xi_{2} \\
\xi_{2} & -\xi_{1}
\end{array}\right)
$$

a deux valeurs propres distinctes qui dégénèrent en une valeur propre double pour $\xi=0$.

Ce modèle est le linéarisé en 0 du modèle générique d'une matrice symétrique réelle d'ordre 2 et de trace nulle, dépendant de deux paramètres réels, et présentant le même phénomène de croisement (voir par exemple [13]). Nous renvoyons à la fin de cette introduction pour des commentaires sur d'éventuelles généralisations de nos résultats au cas générique.

Soit $V: \mathbb{R}^{2} \rightarrow \mathbb{R}$ une fonction de classe $C^{\infty}$. Alors, pour tout réel $h>0$, l'opérateur différentiel

$$
H^{h}=A\left(h D_{x}\right)+V(x)
$$

est essentiellement autoadjoint sur l'espace $\mathcal{H}=L^{2}\left(\mathbb{R}^{2}, \mathbb{C}^{2}\right)$. Pour toute famille $\left(\psi_{0}^{h}\right)$ bornée dans $\mathcal{H}$ quand $h$ tend vers 0 , on se propose d'étudier le comportement, quand $h$ tend vers 0 , de la solution $\psi^{h} \in \mathcal{C}(\mathbb{R}, \mathcal{H})$ du système

$$
i h \partial_{t} \psi^{h}=H^{h} \psi^{h}, \quad \psi^{h}(0)=\psi_{0}^{h} .
$$

Dans le cas particulier où $\psi_{0}^{h}(x)$ a une expression explicite de type état cohérent, une formule approchée pour $\psi^{h}$ a été établie en détail dans les travaux de Hagedorn [12], [13] et Hagedorn-Joye [14]. Ici, nous souhaitons obtenir des informations pour des familles $\left(\psi_{0}^{h}\right)$ générales, satisfaisant seulement à la condition suivante, dite de $h$-oscillation dans [10],

$$
\limsup _{h \rightarrow 0} \int_{h|\xi| \geq R}\left|\widehat{\psi_{0}^{h}}(\xi)\right|^{2} \mathrm{~d} \xi \underset{R \rightarrow \infty}{\longrightarrow} 0
$$

qui correspond intuitivement au fait que les fréquences de Fourier de $\psi_{0}^{h}$ ne dépassent pas l'ordre de $1 / h$.

Dans ce contexte, il est naturel de chercher à déterminer l'évolution des mesures semi-classiques $\mu=\mu(t, x, \tau, \xi)$ de la famille $\left(\psi^{h}\right)$, définies comme les

TOME $130-2002-\mathrm{N}^{\mathrm{O}} 1$ 
valeurs d'adhérence dans $\mathcal{S}^{\prime}\left(\mathbb{R}_{t} \times \mathbb{R}_{x}^{2} \times \mathbb{R}_{\tau} \times \mathbb{R}_{\xi}^{2}\right)$, quand $h$ tend vers 0 , des transformées de Wigner de $\psi^{h}$,

$$
\begin{array}{r}
W^{h} \psi^{h}(t, x, \tau, \xi)=\int_{\mathbb{R} \times \mathbb{R}^{2}} \mathrm{e}^{i(s \tau+y \cdot \xi)} \psi^{h}\left(t-h \frac{s}{2}, x-h \frac{y}{2}\right) \\
\otimes \bar{\psi}^{h}\left(t+h \frac{s}{2}, x+h \frac{y}{2}\right) \frac{\mathrm{d} y \mathrm{~d} s}{(2 \pi)^{3}},
\end{array}
$$

qui sont des mesures positives à valeurs dans les matrices $2 \times 2$ hermitiennes (voir par exemple [10], [11], [21]), et décrivent, dans l'espace des phases, la localisation et la polarisation des particules classiques.

En dehors de $\{\xi=0\}$, le système (3) est à caractéristiques simples, et les résultats de [11] permettent d'obtenir la formule

$$
\mu=\mu^{+} \Pi^{+}+\mu^{-} \Pi^{-},
$$

où les $\Pi^{ \pm}$sont les projecteurs associés à $A(\xi)$,

$$
\Pi^{ \pm}(\xi)=\frac{1}{2}\left(1 \pm \frac{A(\xi)}{|\xi|}\right)
$$

tandis que $\mu^{ \pm}$sont des mesures positives scalaires satisfaisant aux équations de transport

$$
\partial_{t} \mu^{ \pm} \pm \frac{\xi}{|\xi|} \cdot \nabla_{x} \mu^{ \pm}-\nabla V(x) \cdot \nabla_{\xi} \mu^{ \pm}=0
$$

et aux conditions initiales

$$
\mu^{ \pm}(0, x, \tau, \xi)=\operatorname{tr}\left(\Pi^{ \pm} \mu_{0}\right) \delta(\tau \pm|\xi|+V(x))
$$

$\mu_{0}$ désignant une mesure semi-classique de la famille $\left(\psi_{0}^{h}\right)$.

Ainsi, si la mesure $\mu_{0}$ ne charge pas le lieu singulier $\{\xi=0\}$, la mesure $\mu$ est entièrement déterminée par $\mu_{0}$ tant que les courbes caractéristiques des équations (8) — c'est-à-dire les trajectoires classiques — issues du support de $\mu_{0}$ n'atteignent pas $\{\xi=0\}$. L'objectif de ce travail est de décrire $\mu$ près de $\{\xi=0\}$ sans cette dernière restriction.

1.2. Comportement des trajectoires classiques et introduction d'une nouvelle échelle. - Il est naturel d'étudier d'abord les trajectoires classiques associées à ce système et atteignant le croisement $\{\xi=0\}$. De telles trajectoires peuvent être aisément décrites dans une zone où le potentiel $V$ n'a pas de point critique. Précisément, si $x \in \mathbb{R}^{2}$ est tel que $\nabla V(x) \neq 0$, on montre qu'il existe deux courbes $s \mapsto\left(x_{s}^{ \pm}, \xi_{s}^{ \pm}\right)$uniques, continues pour $s$ dans un voisinage de 0 et de classe $C^{1}$ pour $s \neq 0$, telles que

$$
\dot{x}_{s}^{ \pm}= \pm \frac{\xi_{s}^{ \pm}}{\left|\xi_{s}^{ \pm}\right|}, \quad \dot{\xi}_{s}^{ \pm}=-\nabla V\left(x_{s}^{ \pm}\right)
$$

avec les conditions initiales $x_{0}^{ \pm}=x, \xi_{0}^{ \pm}=0$.

BULletin DE LA SOCIÉtÉ MATHÉmATIQUE DE FRANCE 
Sur la trajectoire associée à un mode, on remarque que la quantité $\dot{x}$ change de signe lorsque $\xi$ passe par la valeur 0 . En revanche, la portion future $(s \geq 0)$ de la trajectoire associée à un mode se raccorde de façon régulière à la portion passée $(s \leq 0)$ de la trajectoire associée à l'autre mode (c'est d'ailleurs un fait que l'on retrouve dans le modèle générique, voir le $\S 1.6)$.

En résumé, une particule classique atteignant $\{\xi=0\}$ en un tel point $x$ a le choix entre rester sur cette trajectoire, suivre la trajectoire associée à l'autre mode, ou rester en $\{\xi=0\}$. On peut montrer que cette dernière possibilité n'a jamais lieu si $\nabla V(x) \neq 0$, au sens où la mesure semi-classique de $\left(\psi^{h}\right)$ ne charge pas l'ensemble singulier $\{\xi=0\}$ (cf. $\S 4)$. Nous allons voir que le choix entre les deux possibilités restantes se traduit par un branchement de la mesure semi-classique $\mu$, que nous allons décrire à l'aide d'objets plus précis. L'examen du cas particulier d'un potentiel linéaire va suggérer la nature de ces objets.

Supposons donc pour un moment $V(x)=x_{1}$, et, pour fixer les idées, plaçons-nous dans le cas où les particules classiques atteignent le lieu singulier $\{\xi=0\}$ au même instant $t=t_{0}$. Les trajectoires classiques passant par $\left(x_{0}, 0\right)$ à $t=t_{0}$ sont

$$
x_{t}^{ \pm}=x_{0}+\left(\mp\left|t_{0}-t\right|, 0\right), \quad \xi_{t}^{ \pm}=\left(t_{0}-t, 0\right) .
$$

Les projecteurs spectraux le long de ces courbes vérifient

$$
\begin{aligned}
& \Pi^{+}(t)=\left(\begin{array}{ll}
1 & 0 \\
0 & 0
\end{array}\right) \text { et } \quad \Pi^{-}(t)=\left(\begin{array}{ll}
0 & 0 \\
0 & 1
\end{array}\right) \text { pour } t<t_{0}, \\
& \Pi^{+}(t)=\left(\begin{array}{ll}
0 & 0 \\
0 & 1
\end{array}\right) \text { et } \quad \Pi^{-}(t)=\left(\begin{array}{ll}
1 & 0 \\
0 & 0
\end{array}\right) \text { pour } t>t_{0} .
\end{aligned}
$$

Par ailleurs, le système (3) s'écrit après transformation de Fourier semiclassique dans la variable d'espace

$$
i h \partial_{t} \widehat{\psi}^{h}=A(\xi) \widehat{\psi}^{h}+i h \partial_{\xi_{1}} \widehat{\psi}^{h} .
$$

Il est alors naturel d'effectuer le changement d'échelle $s=\xi_{1} / \sqrt{h}, \eta=\xi_{2} / \sqrt{h}$ qui permet d'éliminer le paramètre $h$ de l'équation et de se ramener au système d'équations différentielles ordinaires

$$
\frac{1}{i} \partial_{s} u=\left(\begin{array}{rr}
s & \eta \\
\eta & -s
\end{array}\right) u
$$

en posant

$$
u(s, t, \eta)=\widehat{\psi}^{h}\left(t-\xi_{1}, \xi_{1}, \xi_{2}\right) .
$$

Près des trajectoires classiques, on a $\xi_{1} \sim\left(t_{0}-t\right)$ donc $s \sim\left(t_{0}-t\right) / \sqrt{h}$, de sorte que le passage de $t<t_{0}$ à $t>t_{0}$ pour le système (3) correspond à un problème de scattering pour (10) paramétré par $\eta$. Il se trouve que la matrice de scattering pour ce système peut être calculée explicitement en fonction de $\eta$, comme l'avaient déjà remarqué Landau [20] et Zener [29] (voir aussi Joye [18] pour un résultat abstrait dans le cadre de la théorie adiabatique). Observons

TOME $130-2002-\mathrm{N}^{\mathrm{O}} 1$ 
que la quantité $\eta=\xi_{2} / \sqrt{h}$ décrit la concentration à l'échelle $\sqrt{h}$ de la transformée de Wigner sur l'ensemble des trajectoires classiques rencontrant $\xi=0$, puisque celui-ci est inclus dans l'hypersurface $I=\left\{\xi_{2}=0\right\}$. Ces remarques font apparaître l'importance du rôle joué par la nouvelle échelle caractéristique $\sqrt{h}$. Comme on va le voir, cette échelle joue un rôle analogue dans le cas d'un potentiel quelconque. Nous sommes conduits à décrire la concentration de la transformation de Wigner sur des sous-variétés involutives de l'espace cotangent, à l'échelle $\sqrt{h}$ : c'est l'objet des mesures semi-classiques à deux échelles.

1.3. Mesures semi-classiques à deux échelles. — Soit $\left(u^{h}\right)$ une famille bornée de $L^{2}\left(\mathbb{R}^{d}\right)$, notons

$$
W^{h} u^{h}(x, \xi)=\int_{\mathbb{R}^{d}} \mathrm{e}^{i y \cdot \xi} u^{h}\left(x-h \frac{y}{2}\right) \bar{u}^{h}\left(x+h \frac{y}{2}\right) \frac{\mathrm{d} y}{(2 \pi)^{d}}
$$

la transformée de Wigner de $u^{h}$, et $\mu$ la mesure semi-classique de $\left(u^{h}\right)$. Rappelons que, si $a \in C_{0}^{\infty}\left(\mathbb{R}^{d} \times \mathbb{R}^{d}\right)$,

$$
\int_{\mathbb{R}^{d} \times \mathbb{R}^{d}} W^{h} u^{h}(x, \xi) a(x, \xi) \mathrm{d} x \mathrm{~d} \xi=\left(\operatorname{op}_{h}(a) u^{h} \mid u^{h}\right),
$$

où $(\mid)$ désigne le produit scalaire sur $L^{2}\left(\mathbb{R}^{d}\right)$ et $\operatorname{op}_{h}(a)$ est le quantifié de Weyl associé au symbole $a$.

Soient $p \in[1, d]$ un entier et $g=\left(g_{1}, \ldots, g_{p}\right): M=T^{*} \mathbb{R}^{d} \rightarrow \mathbb{R}^{p}$ une application de classe $C^{\infty}$ telle que $\mathrm{d} g_{1}, \ldots, \mathrm{d} g_{p}$ soient indépendantes sur $I=\{g=0\}$, et telle que $\left\{g_{i}, g_{j}\right\}=0$ pour tous $i, j$. On désigne par $\mathcal{A}$ l'espace des fonctions $a=a(x, \xi, \eta)$ de classe $C^{\infty}$ sur $M \times \mathbb{R}_{\eta}^{p}$, à support dans $K \times \mathbb{R}_{\eta}^{p}$, où $K$ est une partie compacte de $M$, et telles qu'il existe une fonction $a_{\infty}=a_{\infty}(x, \xi, \omega)$ sur $M \times S^{p-1}$ vérifiant

$$
a(x, \xi, \eta)=a_{\infty}\left(x, \xi, \frac{\eta}{|\eta|}\right)
$$

si $|\eta| \geq R$ pour un certain $R=R(a) \in \mathbb{R}^{+}$. Par ailleurs, on note $\overline{\mathbb{R}^{p}}$ le compactifié de $\mathbb{R}^{p}$ obtenu en ajoutant une sphère à l'infini, et on prolonge $a$ à $\overline{\mathbb{R}^{p}}$ par continuité.

Le théorème suivant est démontré au paragraphe 2.

THÉORÈme 1 . - Il existe une suite $\left(h_{k}\right)$ tendant vers 0 et une mesure positive $\nu_{g}$ sur $I \times \overline{\mathbb{R}^{p}}$ telles que, pour tout $a \in \mathcal{A}$, on ait

$$
\begin{aligned}
& \int_{\mathbb{R}^{d} \times \mathbb{R}^{d}} W^{h} u^{h}(x, \xi) a\left(x, \xi, \frac{g(x, \xi)}{\sqrt{h}}\right) \mathrm{d} x \mathrm{~d} \xi \\
& \underset{\substack{h \rightarrow 0 \\
h=h_{k}}}{\longrightarrow} \int_{I \times \overline{\mathbb{R}^{p}}} a(x, \xi, \eta) \mathrm{d} \nu_{g}(x, \xi, \eta)+\int_{M \backslash I} a_{\infty}\left(x, \xi, \frac{g(x, \xi)}{|g(x, \xi)|}\right) \mathrm{d} \mu(x, \xi) .
\end{aligned}
$$

BULletin DE LA SOCIÉtÉ MATHÉMATIQUE DE FRANCE 
Remarques. - 1) Dans le cas où $g$ est linéaire (concentration sur un sousespace vectoriel involutif), le théorème 1 est dû à Miller [23].

2) Dans un souci de simplicité, nous nous sommes limités ici au cas d'une suite de fonctions à valeurs scalaires, de sorte que $\nu_{g}$ est une mesure à valeurs scalaires. Le théorème 1 s'étend bien sûr au cas d'une suite de fonctions à valeurs vectorielles, $\nu_{g}$ étant alors à valeurs matricielles.

Il est commode de représenter la mesure $\nu_{g}$ de façon plus géométrique, en termes de la sous-variété $I=\{g=0\}$ seulement. Lorsque $m$ varie dans $I$, les espaces vectoriels

$$
N_{m}(I)=T_{m}(M) / T_{m}(I)
$$

s'organisent en un fibré au-dessus de $I$, appelé fibré normal à $I$. En compactifiant chaque fibre par une sphère à l'infini, on obtient un fibré en boules au-dessus de $I$, que nous noterons $\bar{N}(I)$. Alors l'isomorphisme

$$
N_{g}:(m,[v]) \in N(I) \longmapsto(m, \mathrm{~d} g(m) \cdot v) \in I \times \mathbb{R}^{p}
$$

se prolonge par continuité en un isomorphisme de $\bar{N}(I)$ sur $I \times \overline{\mathbb{R}^{p}}$, que nous noterons $\bar{N}_{g}$. On vérifie aisément que l'image réciproque $\nu_{I}$ de $\nu_{g}$ par $\bar{N}_{g}$ dépend seulement de $I$, et non de l'équation $g$.

De plus, on peut montrer l'invariance de $\nu_{I}$ par transformation canonique en un sens convenable.

Enfin, on montre également que, si $\left(u^{h}\right)$ est solution d'une équation scalaire et si $J$ est une sous-variété involutive de l'ensemble caractéristique $\Sigma$ de cette équation, alors la mesure $\nu_{J}$ est supportée dans $\bar{N}_{\Sigma}(J)$, le fibré au-dessus de $\Sigma$ obtenu par compactification des fibres de $T(\Sigma) / T(J)$, et est propagée par le linéarisé transversalement à $J$ du flot hamiltonien associé au symbole principal de l'équation.

Nous renvoyons au paragraphe 2 ci-dessous pour des énoncés précis de ces faits et leurs démonstrations.

1.4. Une formule de Landau-Zener. - Revenons au système (3). Désignons par $S \subset M=T^{*}\left(\mathbb{R}_{t} \times \mathbb{R}_{x}^{2}\right)$ le lieu singulier,

$$
S=\{\xi=0, \tau+V(x)=0\},
$$

qui est l'intersection de la variété caractéristique $\Sigma=\left\{(\tau+V(x))^{2}=|\xi|^{2}\right\}$ avec le lieu du croisement $\{\xi=0\}$. Fixons un point $m_{0}=\left(t_{0}, x_{0},-V\left(x_{0}\right), 0\right) \in S$, et considérons, pour chacun des deux modes, l'union des trajectoires classiques issues d'un point de $S$ au voisinage de $m_{0}$. Notons $J^{ \pm, p}$ (resp. $J^{ \pm, f}$ ) l'ensemble des points du type $\left(t+s, x_{s}^{ \pm}, \tau, \xi_{s}^{ \pm}\right)$où $m=(t, x, \tau, 0)$ décrit un voisinage de $m_{0}$ dans $S$, et $\left.\left.s \in\right]-\epsilon, 0\right]$ (resp. $s \in[0, \epsilon[$ ), où $\epsilon$ est assez petit. Le fait que $\nabla V\left(x_{0}\right) \neq 0$ assure que $J^{ \pm, p}$ et $J^{ \pm, f}$ sont des sous-variétés de codimension 2 dont le bord commun est $S$. De plus, on vérifie que $J^{+, p}, J^{-, f}$ d'une part et

TOME $130-2002-\mathrm{N}^{\mathrm{O}} 1$ 
$J^{-, p}, J^{+, f}$ d'autre part se recollent en des sous-variétés $\mathcal{C}^{\infty}$ sans bord que nous noterons $J, J^{\prime}$, et qui se coupent proprement en $S$, suivant la figure 1 .

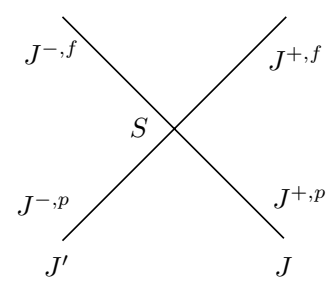

FiguRe 1

On vérifie aisément que $J$ et $J^{\prime}$ sont des sous-variétés involutives de codimension 2. On peut donc considérer les mesures à deux échelles $\nu, \nu^{\prime}$ de la famille $\left(\psi^{h}\right)$ relativement à $J, J^{\prime}$ respectivement. En utilisant le système (3) vérifié par $\psi^{h}$ et le fait que $\mu$ ne charge pas $S$, on aboutit au résultat suivant, où l'on a noté

$$
\begin{gathered}
\Sigma^{+}=\{\tau+V(x)+|\xi|=0, \xi \neq 0\}, \\
\Sigma^{-}=\{\tau+V(x)-|\xi|=0, \xi \neq 0\}, \\
\dot{J}^{ \pm, p}=J^{ \pm, p} \backslash S, \quad \dot{J}^{ \pm, f}=J^{ \pm, f} \backslash S .
\end{gathered}
$$

ThÉORÈme 2. - Il existe des mesures scalaires positives bornées $\nu^{ \pm, p}, \nu^{ \pm, f}$ sur $\bar{N}_{\Sigma^{ \pm}}\left(\dot{J}^{ \pm, p}\right), \bar{N}_{\Sigma^{ \pm}}\left(\dot{J}^{ \pm, f}\right)$ respectivement telles que

$$
\nu=\nu^{+, p} \Pi^{+} \mathbf{1}_{J^{+, p}}+\nu^{-, f} \Pi^{-} \mathbf{1}_{J^{-, f}}, \quad \nu^{\prime}=\nu^{-, p} \Pi^{-} \mathbf{1}_{J^{-, p}}+\nu^{+, f} \Pi^{+} \mathbf{1}_{J^{+, f}} .
$$

De plus, $\nu^{ \pm, p}, \nu^{ \pm, f}$ sont invariantes par le linéarisé du flot hamiltonien de $\tau \pm$ $|\xi|+V(x)$ transversalement à $J^{ \pm, p}, J^{ \pm, f}$ respectivement, dans $\Sigma^{ \pm}$.

Bien sûr, les propriétés d'invariances énoncées dans le théorème 2 s'expriment en coordonnées locales par des équations de transport transverses à $S$. Nous renvoyons au paragraphe 5 pour l'écriture explicite de ces équations. Quoi qu'il en soit, il en résulte que les mesures $\nu^{ \pm, p}, \nu^{ \pm, f}$ admettent, au sens des distributions, des traces au-dessus de $S$ que nous noterons $\nu_{S}^{ \pm, p}, \nu_{S}^{ \pm, f}$. Le phénomène de branchement sera décrit si nous parvenons à relier les deux mesures $\nu_{S}^{ \pm, f}$ aux deux mesures $\nu_{S}^{ \pm, p}$.

Une difficulté préliminaire est que ces mesures ne vivent pas naturellement sur les mêmes espaces. En effet, notons

$$
H=\left(1, \frac{\nabla V}{|\nabla V|}, 0,-\nabla V\right) \quad \text { et } \quad H^{\prime}=\left(1,-\frac{\nabla V}{|\nabla V|}, 0,-\nabla V\right)
$$

les limites des deux champs hamiltoniens le long de $J, J^{\prime}$ au-dessus de $S$, et $H^{\perp}$, $H^{\prime \perp}$ les hyperplans orthogonaux correspondants pour la forme symplectique. 
En étudiant les limites des fibres de $N_{\Sigma^{ \pm}}\left(\dot{J}^{ \pm, p}\right), N_{\Sigma^{ \pm}}\left(\dot{J}^{ \pm, f}\right)$ au-dessus d'un point tendant vers $S$, on constate que $\nu_{S}^{+, p}$ et $\nu_{S}^{-}, f$ sont des mesures sur le compactifié de $H^{\perp} / T J$, tandis que $\nu_{S}^{-, p}, \nu_{S}^{+, f}$ sont des mesures sur le compactifié de $H^{\prime \perp} / T J^{\prime}$. Comme $T J=T S \oplus \mathbb{R} H$ et $T J^{\prime}=T S \oplus \mathbb{R} H^{\prime}$ s'intersectent en $T S$, il est alors naturel d'introduire l'hyperplan

$$
P=T J+T J^{\prime}=T S \oplus \mathbb{R} H \oplus \mathbb{R} H^{\prime},
$$

de sorte que les droites $H^{\perp} / T J$ et $H^{\prime \perp} / T J^{\prime}$ s'identifient toutes deux naturellement à $T M / P$. Pour énoncer notre résultat, il reste à introduire une coordonnée sur cette dernière droite. Un calcul élémentaire montre que $P=\mathbb{R}^{4} \times \mathbb{R} \nabla V$, de sorte que l'on peut repérer la classe modulo $P$ du vecteur tangent $(\delta t, \delta x, \delta \tau, \delta \xi)$ par la coordonnée

$$
\eta=\delta \xi \wedge \frac{\nabla V}{|\nabla V|}
$$

Notre résultat principal est alors :

THÉORÈme 3. - Compte tenu des identifications ci-dessus, on suppose que $\nu_{S}^{+, p}$ et $\nu_{S}^{-, p}$ sont étrangères sur l'ensemble $\{|\eta|<\infty\}$. Alors

$$
\left(\begin{array}{c}
\nu_{S}^{+, f} \\
\nu_{S}^{-}, f
\end{array}\right)=\left(\begin{array}{cc}
1-T & T \\
T & 1-T
\end{array}\right)\left(\begin{array}{l}
\nu_{S}^{+, p} \\
\nu_{S}^{-}, p
\end{array}\right)
$$

où $T(x, \eta)=\mathrm{e}^{-\pi \eta^{2} /|\nabla V(x)|}$.

L'hypothèse selon laquelle les mesures incidentes $\nu_{S}^{ \pm, p}$ sont étrangères évite les interférences entre les deux modes, qui rendraient le problème mal posé en les inconnues $\nu_{S}^{ \pm}$. Un phénomène analogue a été mis en évidence par Nier [25] puis Miller [24] dans les problèmes d'interface.

1.5. Plan de l'article.- Au paragraphe 2, nous introduisons les mesures semi-classiques à deux échelles associées à une sous-variété involutive et établissons les propriétés d'invariance par transformation canonique, de localisation et de propagation qui seront utiles dans la suite.

Le paragraphe 3 est consacré à la géométrie des trajectoires classiques près du croisement et à l'étude des variétés $J$ et $J^{\prime}$. Dans le même esprit qui nous a conduits à introduire l'hyperplan $P$ dans l'équation (12) ci-dessus, on introduit alors une hypersurface $I$ de $M$ telle que $I \cap \Sigma=J \cup J^{\prime}$ (on a d'ailleurs $T I=P$ au-dessus de $S$ ). Compte tenu des résultats du paragraphe 2, la description des mesures $\nu, \nu^{\prime}$ relatives à $J, J^{\prime}$ découlera alors de la description de la mesure $\nu_{I}$ relative à $I$.

Le paragraphe 4 établit que la mesure semi-classique ne charge pas le lieu $S$, ce qui permet de décomposer $\nu_{I}$ suivant les deux modes.

Le paragraphe 5 étudie la propagation des mesures à deux échelles le long de $J, J^{\prime}$ et en dehors de $S$ (théorème 2 ci-dessus).

TOME $130-2002-\mathrm{N}^{\mathrm{O}} 1$ 
Les paragraphes 6,7 et 8 sont consacrés à la démonstration du théorème 3 ci-dessus. Au paragraphe 6 , au moyen d'une transformation canonique, nous ramenons le système 3 au système suivant

$$
\mathrm{op}_{h}(Q) v^{h}=o(h) \quad \text { avec } \quad Q(s, z, \sigma, \zeta)=\left(\begin{array}{cc}
-\sigma+s & \alpha \zeta_{2} \\
\alpha \zeta_{2} & -\sigma-s
\end{array}\right),
$$

où $\alpha=\alpha(\sigma, z)$ est une fonction $\mathcal{C}^{\infty}$ ne s'annulant pas et l'hypersurface correspondant à $I$ est $\left\{\zeta_{2}=0\right\}$.

Au paragraphe 7 , on établit la formule (13) sur le domaine $\{|\eta|=\infty\}$, grâce à une estimation d'énergie hyperbolique pour le système (14).

Enfin, on montre au paragraphe 8, dans l'esprit de [1], [19], [28], [5], que le système (14) admet sur $\{|\eta|<\infty\}$ la forme normale 2-microlocale correspondant à $\alpha=$ Cte, ce qui nous ramène au problème original de Landau-Zener rencontré dans le cas du potentiel linéaire. Un court appendice propose une résolution de ce problème par une méthode d'intégrales oscillantes.

1.6. Remarques sur d'éventuelles généralisations. - Remarquons tout d'abord que la méthode que nous développons dans cet article s'adapte sans difficulté aux linéarisés des matrices présentant des croisements de modes étudiées dans [13]. On peut espérer se libérer de l'hypothèse de linéarité en $\xi$ de ces matrices dès que l'on saura résoudre le cas générique en dimension 2 , c'est-à-dire travailler avec la matrice

$$
A(\xi)=\left(\begin{array}{rr}
p_{1}(\xi) & p_{2}(\xi) \\
p_{2}(\xi) & -p_{1}(\xi)
\end{array}\right),
$$

où $p$ est un difféomorphisme s'annulant en $\xi=0$. Dans ce cas, la géométrie du croisement a beaucoup de similitude avec le cas que nous avons étudié : on peut montrer l'existence de trajectoires classiques rencontrant le lieu singulier $\xi=0$, construire les sous-variétés involutives lisses $J$ et $J^{\prime}$. Il est donc légitime d'espérer qu'une formule de Landau-Zener existe pour ce système et s'énonce en les mêmes termes qu'au théorème 3 ci-dessus. Il ne s'agit pour l'instant que d'une conjecture : bien qu'il existe une hypersurface $I$ donnant un bon cadre de travail, la partie analytique de la preuve qui repose sur l'existence d'une transformation canonique convenable reste pour le moment hors de notre atteinte.

\section{Mesures semi-classiques à deux échelles}

Dans ce paragraphe, nous démontrons le théorème 1 et établissons les propriétés des mesures semi-classiques à deux échelles annoncées dans l'introduction. Considérons une sous-variété involutive $I$ de l'espace cotangent $M=T^{*} \mathbb{R}_{x}^{d}$. Supposons $I$ de codimension $p$ et donnée par

$$
I=\{m=(x, \xi) \in M, g(x, \xi)=0\}
$$

BULletin DE LA SOCiÉtÉ MATHÉmATiQUe DE FRANCE 
où $g=\left(g_{1}, \ldots, g_{p}\right)$ est de classe $\mathcal{C}^{\infty}$ avec $\mathrm{d} g_{1}, \ldots, \mathrm{d} g_{p}$ indépendantes sur $I$ et $\left\{g_{i}, g_{j}\right\}=0$ pour $i \neq j$. Soit $\left(u^{h}\right)$ une suite bornée de $L^{2}\left(\mathbb{R}^{d}\right)$ et $a \in \mathcal{A}$.

La quantité $K_{g}^{h}(a)$ définie par

$$
K_{g}^{h}(a)=\int_{\mathbb{R}^{d} \times \mathbb{R}^{d}} \mathrm{~W}^{h} u^{h}(x, \xi) a\left(x, \xi, \frac{g(x, \xi)}{\sqrt{h}}\right) \mathrm{d} x \mathrm{~d} \xi
$$

s'écrit aussi

$$
K_{g}^{h}(a)=\left(\operatorname{op}_{h}\left(a\left(x, \xi, \frac{g(x, \xi)}{\sqrt{h}}\right)\right) u^{h} \mid u^{h}\right)
$$

En notant $T^{h}$ l'opérateur de changement d'échelle,

$$
T^{h}: f \longmapsto T^{h} f, \quad T^{h} f(x)=\sqrt{h}^{\frac{1}{2} d} f(x \sqrt{h}),
$$

on obtient

$$
K_{g}^{h}(a)=\left(\operatorname{op}_{1}\left(a\left(x \sqrt{h}, \xi \sqrt{h}, \frac{g(x \sqrt{h}, \xi \sqrt{h})}{\sqrt{h}}\right)\right) T^{h} u^{h} \mid T^{h} u^{h}\right) .
$$

La fonction $(x, \xi) \mapsto a(x \sqrt{h}, \xi \sqrt{h}, g(x \sqrt{h}, \xi \sqrt{h}) / \sqrt{h})$ a toutes ses dérivées uniformément bornées en $h$. La suite $\left(T^{h} u_{h}\right)$ étant uniformément bornée en $h$ dans $L^{2}\left(\mathbb{R}^{d}\right)$, il résulte du théorème de Calderon-Vaillancourt ( $c f$. [3] ou [17]) que $K_{g}^{h}(a)$ est uniformément bornée en $h$.

Remarque. - Notons l'importance de la taille de la seconde échelle; on obtiendrait le même résultat en remplaçant $\sqrt{h}$ par $\epsilon(h)$ à condition que $\epsilon(h) \geq C \sqrt{h}$, en revanche le résultat est faux si $\epsilon(h) \ll \sqrt{h}$.

La quantité $K_{g}^{h}(a)$ étant uniformément bornée en $h$, on peut en extraire une suite convergente, puis en considérant une partie dense de $\mathcal{A}$, on construit par un procédé d'extraction diagonal une suite $h_{k}$ tendant vers 0 lorsque $k$ tend vers $+\infty$, telle que $K_{g}^{h_{k}}(a)$ ait une limite $K_{g}(a)$ pour tout $a \in \mathcal{A}$ lorsque $k$ tend vers $+\infty$. Notre but est de montrer que la forme linéaire $K_{g}$ ainsi obtenue s'écrit

$$
K_{g}(a)=\left\langle a, \mu(x, \xi) \mathbf{1}_{(x, \xi) \notin I} \delta\left(\eta-\frac{g(x, \xi)}{|g(x, \xi)|} \infty\right)+\nu(x, \xi, \eta) \mathbf{1}_{(x, \xi) \in I}\right\rangle,
$$

où $\mu$ est la mesure semi-classique de la suite $\left(u^{h_{k}}\right)$ et $\nu$ est une mesure sur $I \times \overline{\mathbb{R}}^{p}$.

Le cas où $I$ est un sous-espace vectoriel $\left\{\xi_{1}=\cdots=\xi_{p}=0\right\}$ est un résultat de L. Miller [23] dont nous rappelons la démonstration ci-dessous. Nous nous ramenons à cette situation au moyen d'une transformation canonique $\chi$ permettant de redresser $I$ en $\left\{\xi_{1}=\cdots=\xi_{p}=0\right\}$ ainsi que d'un opérateur intégral de Fourier semi-classique $U$ associé à $\chi$. L'étude de la concentration sur $I$ d'une suite bornée de $L^{2}\left(\mathbb{R}^{d}\right),\left(v^{h}\right)$, se ramène alors à celle de la suite $\left(U v^{h}\right) \operatorname{sur}\left\{\xi_{1}=\cdots=\xi_{p}=0\right\}$. 


\subsection{Mesures à deux échelles dans le cas d'un sous-espace vectoriel}

Dans ce paragraphe, on suppose que

$$
g(x, \xi)=\xi^{\prime}=\left(\xi_{1}, \ldots, \xi_{p}\right) .
$$

Pour tout $a \in \mathcal{A}$,

$$
\mathrm{op}_{h}^{(2)}(a)=\mathrm{op}_{h}\left(a\left(x, \xi, \frac{\xi^{\prime}}{\sqrt{h}}\right)\right)=\mathrm{op}_{1}\left(a\left(x, h \xi, \sqrt{h} \xi^{\prime}\right)\right)
$$

est un opérateur pseudodifférentiel dont le symbole de Weyl est borné dans $S\left(1, g_{h}\right)$, où la métrique $g_{h}$ est donnée par

$$
g_{h}=\mathrm{d} x^{2}+h^{2} \mathrm{~d}\left(\xi^{\prime \prime}\right)^{2}+h \mathrm{~d}\left(\xi^{\prime}\right)^{2}
$$

en notant $\xi=\left(\xi^{\prime}, \xi^{\prime \prime}\right)$. En vertu des résultats de [16], il s'ensuit que, si $a, b \in \mathcal{A}$,

$$
\mathrm{op}_{h}^{(2)}(a) \mathrm{op}_{h}^{(2)}(b)=\mathrm{op}_{h}^{(2)}(a b)+O(\sqrt{h}) .
$$

On peut alors suivre la preuve de [9] pour montrer l'existence d'une mesure de Radon positive $\rho$ sur $\mathbb{R}_{x}^{d} \times \mathbb{R}_{\xi}^{d} \times \overline{\mathbb{R}}$ telle que

$$
K_{g}(a)=\int a \mathrm{~d} \rho .
$$

Notons que, si la fonction $a$ est supportée en dehors de $I$,

$$
a\left(x, \xi, \frac{\xi^{\prime}}{\sqrt{h}}\right)=a\left(x, \xi, \frac{\xi^{\prime}}{\left|\xi^{\prime}\right|} \infty\right)=a_{\infty}\left(x, \xi, \frac{\xi^{\prime}}{\left|\xi^{\prime}\right|}\right)
$$

pour $h$ assez petit, de sorte que, en dehors de $I$,

$$
\rho(x, \xi, \eta)=\mathbf{1}_{\xi^{\prime} \neq 0} \mu(x, \xi) \otimes \delta\left(\eta-\frac{\xi^{\prime}}{\left|\xi^{\prime}\right|} \infty\right) .
$$

On en déduit l'existence d'une mesure positive $\nu$ sur $I \times \overline{\mathbb{R}^{p}}$ telle que

$$
\int a \mathrm{~d} \rho=\int_{\xi^{\prime} \neq 0} a_{\infty}\left(x, \xi, \frac{\xi^{\prime}}{\left|\xi^{\prime}\right|}\right) \mathrm{d} \mu(x, \xi)+\int_{I \times \overline{\mathbb{R}^{p}}} a \mathrm{~d} \nu,
$$

ce qui est le résultat cherché.

2.2. Opérateurs intégraux de Fourier. - Pour toute fonction $\lambda$ sur $M$, on désigne par $H_{\lambda}$ le champ hamiltonien de $\lambda$, défini par

$$
H_{\lambda} f=\{\lambda, f\} .
$$

Soit $\chi$ une transformation canonique locale de $M=T^{*}\left(\mathbb{R}^{d}\right)$. On peut supposer que $\chi(0,0)=(0,0)$.

Lemme 1. - Il existe une famille $\left(\chi_{s}\right), s \in[0,1]$, de transformations canoniques de $M$ dépendant régulièrement du paramètre $s$ et une famille $\lambda(s)$ de fonctions $\mathcal{C}_{0}^{\infty}$ sur $M$ et à valeurs réelles telles que

$$
\frac{\mathrm{d}}{\mathrm{ds}} \chi_{s}=H_{\lambda(s)}\left(\chi_{s}\right)
$$

BULletin DE LA SOCiÉtÉ MATHÉMATiQUE DE FRANCE 
et $\chi_{0}=\mathrm{Id}, \chi_{1}=\chi$ au voisinage de 0 .

Preuve du lemme. - On peut supposer que $\chi$ est définie sur une boule centrée en 0 . Alors la famille $\left(\tilde{\chi}_{s}\right)$ définie par

$$
\left.\left.\tilde{\chi}_{s}(x, \xi)=\frac{1}{s} \chi(s x, s \xi), \text { pour } s \in\right] 0,1\right] \text { et } \tilde{\chi}_{0}(x, \xi)=\mathrm{d} \chi(0)(x, \xi)
$$

permet de connecter $\chi$ à une transformation canonique linéaire. Le groupe linéaire symplectique étant connexe ( $c f$. [15], ch. X, lemme 2.4), on peut ensuite connecter $\tilde{\chi}_{0}$ à l'identité et construire ainsi au voisinage de 0 une famille régulière $\chi_{s}$ qui connecte l'identité à $\chi$. Puisque chaque $\chi_{s}$ est canonique, il existe une famille $\lambda(s)$ de fonctions $\mathcal{C}^{\infty}$ dans un voisinage de 0 telles que l'on ait (17). En tronquant $\lambda(s)$ au voisinage de 0 , et en résolvant l'équation (17), on obtient une nouvelle famille $\chi_{s}$, cette fois globalement définie sur $M$, et qui coïncide avec la précédente au voisinage de 0.

Notons

$$
\Lambda^{h}(s)=\operatorname{op}_{h}(\lambda(s))
$$

et associons à cette famille $\chi_{s}$ la famille d'opérateurs unitaires définie par

$$
i h \frac{\mathrm{d}}{\mathrm{ds}} U(s)=\Lambda^{h}(s) U(s), \quad U(0)=\mathrm{Id} .
$$

Remarquons que pour tout symbole $a_{s} \in \mathcal{C}_{0}^{\infty}\left(\mathbb{R}_{x}^{d} \times \mathbb{R}_{\xi}^{d}\right)$ et dépendant régulièrement de $s$, nous avons

$$
\begin{aligned}
\frac{\mathrm{d}}{\mathrm{ds}}\left(U(s)^{*} \mathrm{op}_{h}\left(a_{s}\right) U(s)\right) & =U(s)^{*}\left(\mathrm{op}_{h}\left(\partial_{s} a_{s}\right)+\frac{i}{h}\left[\Lambda^{h}(s), \mathrm{op}_{h}\left(a_{s}\right)\right]\right) U(s) \\
& =U(s)^{*} \operatorname{op}_{h}\left(\partial_{s} a_{s}+H_{\lambda(s)} a_{s}\right) U(s)+O\left(h^{2}\right)
\end{aligned}
$$

par le calcul de Weyl. On a donc, si $a_{s} \circ \chi_{s}=a \circ \chi_{1}$ est indépendant de $s$,

$$
\forall a \in \mathcal{C}_{0}^{\infty}\left(\mathbb{R}_{x}^{d} \times \mathbb{R}_{\xi}^{d}\right), \quad U(s)^{*} \operatorname{op}_{h}\left(a_{s}\right) U(s)=\operatorname{op}_{h}\left(a \circ \chi_{1}\right)+O\left(h^{2}\right) .
$$

Dans toute la suite, on appellera opérateur intégral de Fourier unitaire associé à $\chi$, l'opérateur $U=U(1)$, de sorte que (19) s'écrit, pour $s=1$,

$$
\forall a \in \mathcal{C}_{0}^{\infty}\left(\mathbb{R}_{x}^{d} \times \mathbb{R}_{\xi}^{d}\right), \quad U^{*} \operatorname{op}_{h}(a) U=\operatorname{op}_{h}(a \circ \chi)+O\left(h^{2}\right) .
$$

Bien sûr, de tels opérateurs peuvent également être définis par des intégrales oscillantes (comme par exemple dans [27]), mais la définition que nous avons adoptée est plus commode lorsqu'on manipule des opérateurs pseudodifférentiels à deux échelles, comme nous allons le faire dans la suite.

TOME $130-2002-\mathrm{N}^{\mathrm{O}} 1$ 
2.3. Un lemme d'invariance. - Soit $\left(\chi_{s}\right), s \in[0,1]$, une famille de transformations canoniques satisfaisant à $(17)$ avec $\chi_{0}=$ Id et $(U(s))$ la famille d'opérateurs associée comme ci-dessus. Soit $\left(g_{s}\right)$ une famille régulière de fonctions de classe $\mathcal{C}^{\infty}$ de $M$ dans $\mathbb{R}^{p}$ vérifiant $\partial_{s}\left(g_{s} \circ \chi_{s}\right)=0$. Le lemme suivant est une généralisation du «lemme d'Egorov » (20) au cadre des opérateurs à deux échelles.

Précisons d'abord quelques notations. Soit $v^{h}(s)=U(s) v_{0}^{h}$ où $\left(v_{0}^{h}\right)$ est une famille uniformément bornée de $L^{2}\left(\mathbb{R}^{d}\right)$ et soit $\left(a_{s}\right)$ une famille régulière d'éléments de $\mathcal{A}$ vérifiant $\partial_{s}\left(a_{s}\left(\chi_{s}(x, \xi), \eta\right)\right)=0$. On note

$$
K_{g_{s}}^{h}\left(a_{s}\right)=\left(\operatorname{op}_{h}\left(a_{s}\left(x, \xi, \frac{g_{s}(x, \xi)}{\sqrt{h}}\right)\right) v^{h}(s) \mid v^{h}(s)\right) .
$$

Lemme 2. - Il existe une suite $h_{k}$ tendant vers zéro lorsque $k$ tend vers l'infini, telle que pour toute famille $\left(a_{s}\right)$ comme ci-dessus, $\lim _{k \rightarrow+\infty} K_{g_{s}}^{h_{k}}\left(a_{s}\right)$ existe et soit indépendante de $s$.

Preuve du lemme 2. - La famille $\left(v^{h}(s)\right)$ vérifie $i h \partial_{s} v^{h}=\Lambda^{h}(s) v^{h}$. On a donc

$$
\begin{aligned}
\frac{\mathrm{d}}{\mathrm{ds}} K_{g_{s}}^{h}\left(a_{s}\right)= & \frac{1}{i h}\left(\left[\operatorname{op}_{h}\left(a_{s}\left(x, \xi, \frac{g_{s}(x, \xi)}{\sqrt{h}}\right)\right), \Lambda^{h}(s)\right] v^{h}(s) \mid v^{h}(s)\right) \\
& +\left(\operatorname{op}_{h}\left(\partial_{s} a_{s}\left(x, \xi, \frac{g_{s}(x, \xi)}{\sqrt{h}}\right)\right) v^{h}(s) \mid v^{h}(s)\right) \\
& +\left(\frac{1}{\sqrt{h}} \operatorname{op}_{h}\left(\partial_{s} g_{s}(x, \xi) \cdot \nabla_{\eta} a_{s}\left(x, \xi, \frac{g_{s}(x, \xi)}{\sqrt{h}}\right)\right) v^{h}(s) \mid v^{h}(s)\right) .
\end{aligned}
$$

Notons

$$
\Theta=\frac{1}{i h}\left[\operatorname{op}_{h}\left(a_{s}\left(x, \xi, \frac{g_{s}(x, \xi)}{\sqrt{h}}\right)\right), \Lambda^{h}(s)\right] .
$$

Si $T^{h}$ est l'opérateur de changement d'échelle défini en (16), alors

$$
T^{h} \Theta\left(T^{h}\right)^{*}=\frac{1}{i h}\left[\operatorname{op}_{1}\left(a_{s}\left(x \sqrt{h}, \xi \sqrt{h}, \frac{g_{s}(x \sqrt{h}, \xi \sqrt{h})}{\sqrt{h}}\right)\right), \mathrm{op}_{1}(\lambda(s, x \sqrt{h}, \xi \sqrt{h}))\right] .
$$

Le lemme de calcul de Weyl suivant est classique (cf. par exemple [16], p. 155) :

Lemme 3. - Soient a et $b$ deux fonctions $\mathcal{C}^{\infty}$ à dérivées bornées; alors

$$
\left[\mathrm{op}_{1}(a), \mathrm{op}_{1}(b)\right]=\mathrm{op}_{1}\left(\frac{1}{i}\{a, b\}+r\right)
$$

où

$$
\mathrm{N}_{p}(r)=\sup _{|\alpha|+|\beta| \leq p}\left|\partial_{x}^{\alpha} \partial_{\xi}^{\beta} r\right| \leq C \sum_{q+q^{\prime}=p}\left(\mathrm{~N}_{q}\left(D^{3} a\right) \mathrm{N}_{q^{\prime}}\left(D^{3} b\right)\right)
$$

BULLETIN DE LA SOCIÉtÉ MATHÉMATIQUE DE FRANCE 
En utilisant ce lemme, on obtient

$$
\begin{array}{r}
T^{h} \Theta\left(T^{h}\right)^{*}=-\frac{1}{h} \operatorname{op}_{1}\left(\left\{a_{s}\left(x \sqrt{h}, \xi \sqrt{h}, \frac{g_{s}(x \sqrt{h}, \xi \sqrt{h})}{\sqrt{h}}\right), \lambda(s, x \sqrt{h}, \xi \sqrt{h})\right\}\right) \\
+O(\sqrt{h})
\end{array}
$$

dans $\mathcal{L}\left(L^{2}\left(\mathbb{R}^{d}\right)\right)$, soit

$$
\begin{aligned}
\Theta=\operatorname{op}_{h}\left(H_{\lambda(s)}\left(a_{s}\right)\right. & \left(x, \xi, \frac{g_{s}(x, \xi)}{\sqrt{h}}\right) \\
& \left.+\frac{1}{\sqrt{h}} H_{\lambda(s)}\left(g_{s}\right) \cdot \nabla_{\eta} a_{s}\left(x, \xi, \frac{g_{s}(x, \xi)}{\sqrt{h}}\right)\right)+O(\sqrt{h}),
\end{aligned}
$$

ce qui, reporté dans $(21)$, donne $\mathrm{d} K_{g_{s}}^{h}\left(a_{s}\right) / \mathrm{d} s=O(\sqrt{h})$, uniformément en $h$. Il suffit alors de choisir la suite $h_{k}$ de sorte que la limite de $K_{g_{0}}^{h_{k}}\left(a_{0}\right)$ existe pour tout $a_{0}$.

\subsection{Définition et nature géométrique des mesures à deux échelles}

Preuve du théorème 1. - Soit $\chi$ une transformation canonique locale satisfaisant à

$$
g \circ \chi^{-1}(x, \xi)=\xi^{\prime}
$$

Par le lemme 1 , on associe à $\chi$ une famille de transformations canoniques $\chi_{s}$. On note $g_{s}$ la famille d'équations définie par $g_{s} \circ \chi_{s}=g$ pour tout $s \in[0,1]$.

Soient $U$ un opérateur intégral de Fourier associé à $\chi$ et $\left(v^{h}\right)$ une suite uniformément bornée dans $L^{2}\left(\mathbb{R}^{d}\right)$ dont nous voulons étudier la concentration sur $I=\{g=0\}$ à l'échelle $\sqrt{h}$. Notons $v_{0}^{h}=v^{h}$, de sorte que, avec les notations du lemme 2, on ait $v^{h}(1)=U v^{h}$. Par ce même lemme, on obtient l'existence d'une suite $h_{k}$ telle que pour tout $a \in \mathcal{A}$

$$
\lim _{k \rightarrow+\infty} K_{g}^{h_{k}}(a)=\lim _{k \rightarrow+\infty} K_{g_{s}}^{h_{k}}\left(a_{s}\right)=\lim _{k \rightarrow+\infty} K_{g_{1}}^{h_{k}}\left(a_{1}\right),
$$

où $a_{s}$ est définie par $a_{s} \circ \chi_{s}=a$. Par le résultat du paragraphe 2.1 , il existe une mesure $\nu_{1}$ sur $\left\{\xi^{\prime}=0\right\} \times \overline{\mathbb{R}^{p}}$ telle que,

$$
\lim _{k \rightarrow+\infty} K_{g_{1}}^{h_{k}}\left(a_{1}\right)=\left\langle a_{1}(x, \xi, \eta), \mu_{1}(x, \xi) \mathbf{1}_{\xi^{\prime} \neq 0} \delta\left(\eta-\frac{\xi^{\prime}}{\left|\xi^{\prime}\right|} \infty\right)+\nu_{1}\left(x, \xi^{\prime \prime}, \eta\right) \delta\left(\xi^{\prime}\right)\right\rangle,
$$

où $\mu_{1}$ est une mesure semi-classique de la suite $\left(v^{h}(1)\right)$. Le théorème 1 est donc démontré en choisissant pour $\nu_{g}$ l'image réciproque de $\nu_{1}$ par l'application $\tilde{\chi}$ définie par $\tilde{\chi}(m, \eta)=(\chi(m), \eta)$.

Notons $\nu_{I}$ la mesure sur $\bar{N}(I)$, image réciproque de $\nu_{g}$ par l'isomorphisme $\bar{N}_{g}$, où $N_{g}(m,[v])=(m, \mathrm{~d} g(m) \cdot v)$. Cette mesure ne dépend pas de

TOME $130-2002-\mathrm{N}^{\mathrm{O}} 1$ 
l'équation $g$ : en effet, soit $\tilde{g}$ une fonction telle que $\tilde{g}=\theta g$, où $\theta$ est une matrice inversible. Alors, d'une part, $\mathrm{d} \tilde{g}=\theta \mathrm{d} g$ sur $I$; d'autre part,

$$
\mathrm{op}_{h}\left(a\left(x, \xi, \frac{\tilde{g}(x, \xi)}{\sqrt{h}}\right)\right)=\operatorname{op}_{h}\left(\tilde{a}\left(x, \xi, \frac{g(x, \xi)}{\sqrt{h}}\right)\right)
$$

avec $\tilde{a}(x, \xi, \eta)=a(x, \xi, \theta(x, \xi) \eta)$. Ainsi $\tilde{a} \circ \bar{N}_{g}=a \circ \bar{N}_{\tilde{g}}$ et $\left\langle\nu_{g}, \tilde{a}\right\rangle=\left\langle\nu_{\tilde{g}}, a\right\rangle$. La mesure $\nu_{I}$ est donc bien définie indépendamment de $g$.

Par construction, la mesure $\nu_{I}$ est invariante par transformation canonique au sens suivant. Considérons une sous-variété involutive $I$ de $M$ et $\chi$ une transformation canonique de $M$. L'application tangente $T \chi$ envoie $T I$ sur $T \chi(I)$, elle induit donc une application $N(\chi)$ de $N(I)$ sur $N(\chi(I))$ que l'on peut prolonger en une application $\bar{N}(\chi)$ de $\bar{N}(I)$ sur $\bar{N}(\chi(I))$ en convenant que si $(m,[v]) \in N(I)$ et $N(\chi)(m,[v])=(\chi(m),[w])$, alors

$$
\bar{N}(\chi)(m,[v] \infty)=(\chi(m),[w] \infty) .
$$

Considérons enfin un opérateur intégral de Fourier $U$ associé à $\chi$. Avec ces notations nous avons la proposition suivante :

Proposition 1. - Soit $\left(v^{h}\right)$ une suite uniformément bornée de $L^{2}\left(\mathbb{R}^{d}\right)$ et $\nu_{I}$ sa mesure à deux échelles pour I. Alors la concentration de $\left(U v^{h}\right)$ sur $\chi(I)$ est décrite par une mesure à deux échelles $\nu_{\chi(I)}$ vérifiant

$$
\nu_{\chi(I)} \circ \bar{N}(\chi)=\nu_{I}
$$

Avant d'envisager le cas de solutions d'une équation aux dérivées partielles, remarquons que comme dans le cas microlocal, le fait que les mesures à deux échelles $\nu$ et $\tilde{\nu}$ de deux suites $\left(u^{h}\right)$ et $\left(\tilde{u}^{h}\right)$ pour la même involutive $I$ soient étrangères permet de calculer la mesure de $\left(u^{h}+\tilde{u}^{h}\right)$. En effet, notons

$$
v^{h}=\left(\begin{array}{c}
u^{h} \\
\tilde{u}^{h}
\end{array}\right) .
$$

La mesure à deux échelles de $\left(v^{h}\right)$ sur $I$ est alors de la forme

$$
\left(\begin{array}{cc}
\nu & \bar{\lambda} \\
\lambda & \tilde{\nu}
\end{array}\right)
$$

et sa positivité entraîne que $\lambda$ est une mesure absolument continue par rapport à $\nu$ et $\tilde{\nu}$. On a donc $\lambda=0$, ce qui implique

$$
\left(\operatorname{op}_{h}\left(a\left(x, \xi, \frac{g(x, \xi)}{\sqrt{h}}\right)\right) u^{h} \mid \tilde{u}^{h}\right) \underset{h \rightarrow 0}{\longrightarrow} 0,
$$

et la mesure de $\left(u^{h}+\tilde{u}^{h}\right)$ n'est autre que $\nu+\tilde{\nu}$. Cette remarque sera utile au paragraphe 8.

BULletin DE LA SOCiÉtÉ MATHÉmATiQUe DE FRANCE 
2.5. Localisation et propagation. - Venons-en aux propriétés de $\nu_{I}$ lorsque $\left(u^{h}\right)$ est solution d'une équation aux dérivées partielles. Soit $q: M \rightarrow \mathbb{R}$ une fonction de classe $C^{\infty}$, et soit $\left(Q^{h}\right)$ la famille d'opérateurs pseudodifférentiels semi-classiques associée à $q$ par la quantification de Weyl,

$$
Q^{h} v(x)=\int_{\mathbb{R}^{d}} \int_{\mathbb{R}^{d}} \mathrm{e}^{i(x-y) \cdot \xi} q\left(\frac{x+y}{2}, h \xi\right) v(y) \frac{\mathrm{d} y \mathrm{~d} \xi}{(2 \pi)^{d}} .
$$

La formule ci-dessus a un sens au moins si $q$ a une croissance polynomiale à l'infini en $\xi$, ce que l'on peut toujours supposer car les propriétés étudiées sont locales en les variables $(x, \xi)$. Soit $m_{0} \in M$ tel que $q\left(m_{0}\right)=0, \mathrm{~d} q\left(m_{0}\right) \neq 0$, et soit $\Sigma$ l'hypersurface d'équation $q=0$ près de $m_{0}$. Soit $\left(u^{h}\right)$ une suite bornée de $L^{2}\left(\mathbb{R}^{d}\right)$ telle que, pour toute fonction $\beta \in C^{\infty}\left(\mathbb{R}^{d} \times \mathbb{R}^{d}\right)$ à support dans un voisinage donné de $m_{0}$, on ait dans $L^{2}\left(\mathbb{R}^{d}\right)$

$$
\operatorname{op}_{h}(\beta) Q^{h} u^{h}=o(h) .
$$

Soit $I=\{g=0\}$ une sous-variété involutive de $T^{*} \mathbb{R}^{d}$. On suppose que $I$ coupe transversalement $\Sigma$. La mesure $\nu_{I}$, comme la mesure semi-classique de la suite $\left(u^{h}\right)$, est supportée au-dessus de $\Sigma$. Supposons de plus que $I \cap \Sigma$ soit involutive. La concentration de $\left(u^{h}\right)$ sur $I$ est alors décrite d'une part par $\nu_{I}$, d'autre part par $\nu_{\Sigma \cap I}$. Le lien entre ces deux mesures est décrit par le lemme suivant. Rappelons que, si $J$ est une sous-variété involutive de $\Sigma$, nous notons $\bar{N}_{\Sigma}(J)$ le fibré au-dessus de $\Sigma$ obtenu par compactification des fibres de $T(\Sigma) / T(J)$. Si $J=I \cap \Sigma$, l'isomorphisme canonique de $T(\Sigma) / T(J)$ sur $T(M)_{\mid J} / T(I)_{\mid J}$ se prolonge en un isomorphisme

$$
\theta_{\Sigma, I}: \bar{N}_{\Sigma}(J) \longrightarrow \overline{N(I)}_{\mid J}
$$

LEMme 4. - Soit $J$ une sous-variété involutive de $\Sigma$, alors $\operatorname{Supp}\left(\nu_{J}\right) \subset$ $\bar{N}_{\Sigma}(J)$. De plus, si $J=I \cap \Sigma$, les mesures $\nu_{I}$ et $\nu_{J}$ sont identifiées par l'isomorphisme $\theta_{\Sigma, I}$.

Preuve du lemme 4. - Compte-tenu de l'invariance par transformation canonique des mesures $\nu_{J}$ et $\nu_{I}$, il suffit de démontrer ce résultat dans un système de coordonnées adaptées. Supposons que

$$
\Sigma=\left\{\xi_{1}=0\right\}, \quad J=\left\{\xi_{1}=\cdots=\xi_{p}=0\right\}, \quad I=\left\{\xi_{2}=\cdots=\xi_{p}=0\right\} .
$$

L'équation (23) devient $i h \partial_{x_{1}} u_{h}=o(h)$, microlocalement. La classe modulo $T J$ d'un élément $\delta \xi=\left(0, \delta \xi_{2}, \ldots, \delta \xi_{d}\right)$ de $T \Sigma$ est repérée par ses $p$ premières coordonnées $\left(\delta \xi_{1}=0, \ldots, \delta \xi_{p}\right)$ et est donc complètement déterminée par la connaissance de $\left(\delta \xi_{2}, \ldots, \delta \xi_{p}\right)$. Soit $a \in \mathcal{A}$ nul sur $\left\{\eta_{1}=0\right\}$. Alors $a$ est de la forme $a(x, \xi, \eta)=\eta_{1} b(x, \xi, \eta)$, avec $b$ de classe $C^{\infty}$ bornée ainsi que ses dérivées.

TOME $130-2002-\mathrm{N}^{\mathrm{O}} 1$ 
Il vient, en utilisant le calcul symbolique puis l'équation,

$$
\begin{aligned}
\left(\operatorname{op}_{h}(\right. & \left.\left.\left(x, \xi, \frac{\left(\xi_{1}, \ldots, \xi_{p}\right)}{\sqrt{h}}\right)\right) u^{h} \mid u^{h}\right) \\
\quad= & \left(\operatorname{op}_{h}\left(b\left(x, \xi, \frac{\left(\xi_{1}, \ldots, \xi_{p}\right)}{\sqrt{h}}\right)\right) \operatorname{op}_{h}\left(\frac{\xi_{1}}{\sqrt{h}}\right) u^{h} \mid u^{h}\right)+O(\sqrt{h})=O(\sqrt{h}) .
\end{aligned}
$$

Le support de $\nu_{J}$ est donc contenu dans $\left\{\eta_{1}=0\right\}$, c'est-à-dire $\bar{N}_{\Sigma}(J)$.

Pour tout $a \in \mathcal{A}, a-a_{\mid \eta_{1}=0}=\eta_{1} b$, où $b$ est comme ci-dessus. Il en résulte que

$$
\left(\mathrm{op}_{h}(a) u^{h} \mid u^{h}\right)=\left(\mathrm{op}_{h}\left(a_{\mid \eta_{1}=0}\right) u^{h} \mid u^{h}\right)+O(\sqrt{h}),
$$

d'où $\left\langle\nu_{J}, a\right\rangle=\left\langle\nu_{I}, a_{\mid \eta_{1}=0}\right\rangle$, ce qu'il fallait démontrer.

Venons-en à l'étude de la propagation de ces mesures et commençons par introduire quelques notations; comme $J=\Sigma \cap I$ est une sous-variété involutive, nous avons

$$
\{q, g\}=c g+q \tilde{c},
$$

où $c \in \mathcal{M}_{p}(\mathbb{R})$, et $\tilde{c} \in \mathbb{R}^{p}$ sont des fonctions à valeurs matricielles et vectorielles respectivement, $C^{\infty}$ près de $m_{0}$.

Proposition 2. - Soit $\left(u^{h}\right)$ une suite bornée de $L^{2}\left(\mathbb{R}^{d}\right)$ satisfaisant à (23), $\nu_{I}$ et $\nu_{J}$ les mesures à deux échelles associées. La mesure $\nu_{J}$ est propagée par la linéarisation du flot hamiltonien de $q$ transversalement à $\Sigma$. Cette propriété se traduit sur la mesure $\nu_{g}=\bar{N}_{g}\left(\nu_{I}\right)$ par l'équation de transport

$$
\left\{q, \nu_{g}\right\}+c \nabla_{\eta} \cdot\left(\eta \nu_{g}\right)=0
$$

où la matrice c est définie par (24).

Notons que l'équation (25) doit se comprendre dans le dual de l'espace $\mathcal{A}$, en tenant compte du fait que $\eta \cdot \nabla_{\eta} a=0$ pour tout $a \in \mathcal{A}$ au voisinage de $|\eta|=+\infty$.

Preuve de la proposition 2. - Nous suivons la même stratégie qu'au lemme 2. Considérons $\beta \in \mathcal{C}^{\infty}\left(\mathbb{R}_{x}^{d} \times \mathbb{R}_{\xi}^{d}\right)$ à support compact près de $m_{0}$ et vérifiant $\beta=1$ près de $m_{0}$ et $\{q, \beta\}=0$ dans l'intérieur $\mathcal{U}$ de $\{\beta=1\}$. Soit $a \in \mathcal{A}$ à support compact en $(x, \xi)$ dans $\mathcal{U}$. Comme pour le lemme 2 , la preuve de la proposition 2 repose sur l'étude de l'opérateur

$$
\tilde{\Theta}=\frac{i}{h} T^{h}\left[\operatorname{op}_{h}(\beta) Q^{h}, \operatorname{op}_{h}\left(a\left(x, \xi, \frac{g(x, \xi)}{\sqrt{h}}\right)\right)\right]\left(T^{h}\right)^{*},
$$

pour lequel on a $\left(\tilde{\Theta} T^{h} u^{h} \mid T^{h} u^{h}\right)=o(1)$ du fait de l'équation (23) à laquelle satisfait $u^{h}$. Or, compte tenu de la localisation des supports de $\beta$ et $a$, on a

$$
\tilde{\Theta}=\frac{i}{h} T^{h}\left[\operatorname{op}_{h}(\beta q), \mathrm{op}_{h}\left(a\left(x, \xi, \frac{g(x, \xi)}{\sqrt{h}}\right)\right)\right]\left(T^{h}\right)^{*}+o(1),
$$

BULletin DE LA SOCiÉtÉ MATHÉMATIQUE DE FRANCE 
dans $\mathcal{L}\left(L^{2}\left(\mathbb{R}^{d}\right)\right)$.

Le lemme 3 (ainsi que la localisation des supports de $\beta$ et de $a$ ) nous donne

$$
\begin{aligned}
\tilde{\Theta}=\mathrm{op}_{1}(\{ & \left.q, a\}\left(x \sqrt{h}, \xi \sqrt{h}, \frac{g(x \sqrt{h}, \xi \sqrt{h})}{\sqrt{h}}\right)\right) \\
& +\mathrm{op}_{1}\left(\frac{1}{\sqrt{h}}\{q, g\} \cdot \nabla_{\eta} a\left(x \sqrt{h}, \xi \sqrt{h}, \frac{g(x \sqrt{h}, \xi \sqrt{h})}{\sqrt{h}}\right)\right)+o(1) .
\end{aligned}
$$

Le premier terme du membre de droite de l'équation ci-dessus donne le premier terme de l'équation de transport (25); il reste donc à étudier le second terme.

Remarquons tout-d'abord que la formule (24) et la localisation du support de $a$ donnent

$$
\begin{aligned}
\mathrm{op}_{1}\left(\frac{1}{\sqrt{h}}\{q, g\} \cdot \nabla_{\eta} a\left(x \sqrt{h}, \xi \sqrt{h}, \frac{g(x \sqrt{h}, \xi \sqrt{h})}{\sqrt{h}}\right)\right) \\
=\mathrm{op}_{1}\left(\frac{c g}{\sqrt{h}} \cdot \nabla_{\eta} a\left(x \sqrt{h}, \xi \sqrt{h}, \frac{g(x \sqrt{h}, \xi \sqrt{h})}{\sqrt{h}}\right)\right) \\
\quad+\frac{1}{\sqrt{h}} \mathrm{op}_{1}\left(\beta q \tilde{c} \cdot \nabla_{\eta} a\left(x \sqrt{h}, \xi \sqrt{h}, \frac{g(x \sqrt{h}, \xi \sqrt{h})}{\sqrt{h}}\right)\right) .
\end{aligned}
$$

Le premier terme du membre de droite de l'égalité ci-dessus donne le second terme de (25). La contribution du deuxième terme est nulle. Pour s'en convaincre, on réutilise l'équation (23) en écrivant

$$
\begin{aligned}
& \frac{1}{\sqrt{h}} \operatorname{op}_{1}\left(\beta q \tilde{c} \cdot \nabla_{\eta} a\left(x \sqrt{h}, \xi \sqrt{h}, \frac{g(x \sqrt{h}, \xi \sqrt{h})}{\sqrt{h}}\right)\right) \\
& =\frac{1}{\sqrt{h}} \operatorname{op}_{1}\left(\tilde{c} \cdot \nabla_{\eta} a\left(x \sqrt{h}, \xi \sqrt{h}, \frac{g(x \sqrt{h}, \xi \sqrt{h})}{\sqrt{h}}\right)\right) \operatorname{op}_{1}(\beta q(x \sqrt{h}, \xi \sqrt{h}))+R^{h},
\end{aligned}
$$

où $R^{h}$ est uniformément borné, et a la même structure que $\sqrt{h} \tilde{\Theta}$. On a donc gagné une puissance de $\sqrt{h}$, ce qui est suffisant pour obtenir

$$
\left(R^{h} T^{h} u^{h} \mid T^{h} u^{h}\right)=O(\sqrt{h})
$$

en reprenant sur $R^{h}$ l'étude faite sur $\tilde{\Theta}$. Ceci clôt la preuve de la proposition 2 .

\section{Géométrie des trajectoires classiques près du croisement.}

Proposition 3. - Soit $x \in \mathbb{R}^{2}$ tel que $\nabla V(x) \neq 0$, il existe deux courbes $s \mapsto\left(x_{s}^{ \pm}, \xi_{s}^{ \pm}\right)$uniques, continues pour $s$ dans un voisinage de 0 et de classe $C^{1}$ pour $s \neq 0$, telles que

$$
\dot{x}_{s}^{ \pm}= \pm \frac{\xi_{s}^{ \pm}}{\left|\xi_{s}^{ \pm}\right|}, \quad \dot{\xi}_{s}^{ \pm}=-\nabla V\left(x_{s}^{ \pm}\right),
$$

TOME $130-2002-\mathrm{N}^{\mathrm{O}} 1$ 
avec les conditions initiales $x_{0}^{ \pm}=x, \xi_{0}^{ \pm}=0$. De plus, $\left(x_{s}^{ \pm}, \xi_{s}^{ \pm}\right)$dépendent de façon $C^{\infty}$ de $(s, x)$ pour $s \neq 0$.

Notons que (26) implique en particulier

$$
\pm \dot{x}_{s=0^{+}}^{ \pm}=\mp \dot{x}_{s=0^{-}}^{ \pm}=-\frac{\nabla V(x)}{|\nabla V(x)|} .
$$

Preuve de la proposition 3. - Soit $x \in \mathbb{R}^{2}$ tel que $\nabla V(x) \neq 0$. Considérons le système

$$
\begin{cases}\dot{X}_{s}=-\operatorname{sgn}(s) \frac{\Xi_{s}}{\left|\Xi_{s}\right|}, & X_{0}=x \\ \dot{\Xi}_{s}=-\nabla V\left(X_{s}\right), & \Xi_{0}=0\end{cases}
$$

Le système (27) équivaut à

$$
\Xi_{s}=-s \int_{0}^{1} \nabla V\left(X_{s t}\right) \mathrm{d} t, \quad X_{s}=x+\int_{0}^{s} \frac{\int_{0}^{1} \nabla V\left(X_{\sigma t}\right) \mathrm{d} t}{\left|\int_{0}^{1} \nabla V\left(X_{\sigma t}\right) \mathrm{d} t\right|} \mathrm{d} \sigma,
$$

l'hypothèse $\nabla V(x) \neq 0$ et le théorème du point fixe nous assurent que le système (27) admet une unique solution dans un voisinage de $s=0$. De plus cette solution dépend de façon $\mathcal{C}^{\infty}$ de $x$. Il en est de même pour le système

$$
\begin{cases}\dot{X}_{s}^{\prime}=\operatorname{sgn}(s) \frac{\Xi_{s}^{\prime}}{\left|\Xi_{s}^{\prime}\right|}, & X_{0}^{\prime}=x \\ \dot{\Xi}_{s}^{\prime}=-\nabla V\left(X_{s}^{\prime}\right), & \Xi_{0}^{\prime}=0 .\end{cases}
$$

On remarque alors que les courbes

$$
\left(x_{s}^{+}, \xi_{s}^{+}\right)=\left\{\begin{array}{l}
\left(X_{s}, \Xi_{s}\right) \text { pour } s \leq 0, \\
\left(X_{s}^{\prime}, \Xi_{s}^{\prime}\right) \text { pour } s \geq 0,
\end{array} \quad\left(x_{s}^{-}, \xi_{s}^{-}\right)=\left\{\begin{array}{l}
\left(X_{s}^{\prime}, \Xi_{s}^{\prime}\right) \text { pour } s \leq 0, \\
\left(X_{s}, \Xi_{s}\right) \text { pour } s \geq 0,
\end{array}\right.\right.
$$

sont solutions de (26). Réciproquement, on construit des solutions de (27) et $(28)$ au moyen de $\left(x_{s}^{+}, \xi_{s}^{+}\right)$et $\left(x_{s}^{-}, \xi_{s}^{-}\right)$, solutions de $(26)$. L'unicité des solutions des deux premières équations implique l'unicité des solutions de (26), ce qui prouve la proposition 3 .

Soit $m_{0}=\left(t_{0}, x_{0}, \tau_{0}, \xi_{0}\right) \in M$ tel que $\xi_{0}=0, \tau_{0}+V\left(x_{0}\right)=0$ et $\nabla V\left(x_{0}\right) \neq 0$. On définit

$$
\begin{aligned}
& \left.\left.J^{+, p}=\left\{\left(t+s, X_{s}(x), \tau, \Xi_{s}(x)\right),(t, x, \tau, 0) \in \mathcal{U}, s \in\right]-\epsilon, 0\right]\right\}, \\
& \left.\left.J^{-, p}=\left\{\left(t+s, X_{s}^{\prime}(x), \tau, \Xi_{s}^{\prime}(x)\right),(t, x, \tau, 0) \in \mathcal{U}, s \in\right]-\epsilon, 0\right]\right\}, \\
& J^{+, f}=\left\{\left(t+s, X_{s}^{\prime}(x), \tau, \Xi_{s}^{\prime}(x)\right),(t, x, \tau, 0) \in \mathcal{U}, s \in[0, \epsilon[\},\right. \\
& J^{-, f}=\left\{\left(t+s, X_{s}(x), \tau, \Xi_{s}(x)\right),(t, x, \tau, 0) \in \mathcal{U}, s \in[0, \epsilon[\},\right.
\end{aligned}
$$

où $\mathcal{U}$ est un voisinage de $m_{0}$ dans $S \cap\{\nabla V(x) \neq 0\}$ (on rappelle que $S$ est défini par $S=\{\xi=0, \tau+V(x)=0\}$ ) et $\epsilon$ est choisi assez petit de sorte que 
l'on reste dans l'ensemble $\{\nabla V(x) \neq 0\}$ et dans le domaine d'existence des solutions de (27) et (28). On a donc

$$
\begin{aligned}
J= & J^{+, p} \cup J^{-, f} \\
=\left\{(t, x, \tau, \xi) ; \exists(s, y) \in \mathbb{R} \times \mathbb{R}^{2},(x, \xi)=\right. & \left(X_{s}(y), \Xi_{s}(y)\right), \\
& (t-s, y, \tau, 0) \in \mathcal{U}\}, \\
J^{\prime}=J^{-, p} \cup J^{+, f} & \\
=\left\{(t, x, \tau, \xi) ; \exists(s, y) \in \mathbb{R} \times \mathbb{R}^{2},(x, \xi)=\right. & \left(X_{s}^{\prime}(y), \Xi_{s}^{\prime}(y)\right), \\
& (t-s, y, \tau, 0) \in \mathcal{U}\} .
\end{aligned}
$$

Compte tenu de la construction de $\left(X_{s}, \Xi_{s}\right)$ et $\left(X_{s}^{\prime}, \Xi_{s}^{\prime}\right)$, $J$ et $J^{\prime}$ sont des sousvariétés $\mathcal{C}^{\infty}$ se coupant transversalement en $S$.

Proposition 4. - Il existe une fonction $\phi$ de classe $\mathcal{C}^{\infty}$ dans un voisinage de $\left(x_{0}, \tau_{0}\right)$ telle que $J=\{\xi=\nabla \phi(x, \tau)\}, J^{\prime}=\{\xi=-\nabla \phi(x, \tau)\}$. En particulier, $J$ et $J^{\prime}$ sont des sous-variétés involutives de $T^{*}\left(\mathbb{R}^{3}\right)$. De plus

$$
|\tau+V(x)|=|\nabla \phi(x, \tau)| \text {. }
$$

Notons que la formule (29) traduit le fait que $J \cup J^{\prime} \subset \Sigma=\{|\tau+V(x)|=|\xi|\}$. On définit alors $\omega=\omega(x, \tau)$ par la formule

$$
\nabla \phi(x, \tau)=(\tau+V(x)) \omega(x, \tau),
$$

et on note

$$
I=\{m=(t, x, \tau, \xi) ; \omega(x, \tau) \wedge \xi=0\},
$$

où pour $v=\left(v_{1}, v_{2}\right) \in \mathbb{R}^{2}$ et $y=\left(y_{1}, y_{2}\right) \in \mathbb{R}^{2}$, on note

$$
v \wedge y=v_{1} y_{2}-v_{2} y_{1}=v^{\perp} \cdot y
$$

avec $v^{\perp}=\left(-v_{2}, v_{1}\right)$.

On vérifie que $I$ est une hypersurface coupant transversalement

$$
\begin{aligned}
& \Sigma^{+}=\{\tau+V(x)+|\xi|=0, \xi \neq 0\} \quad \text { et } \\
& \Sigma^{-}=\{\tau+V(x)-|\xi|=0, \xi \neq 0\},
\end{aligned}
$$

et que $\Sigma \cap I=J \cup J^{\prime}$.

Preuve de la proposition 4. - Pour tout $\tau_{0} \in \mathbb{R}$, considérons les ensembles

$$
\Lambda_{\tau_{0}}=\left\{\left(X_{s}(x), \Xi_{s}(x)\right) ; \exists t \in \mathbb{R},\left(t, x, \tau_{0}, 0\right) \in \mathcal{U}\right\},
$$

$\left(\operatorname{resp} . \Lambda_{\tau_{0}}^{\prime}=\left\{\left(X_{s}^{\prime}(x), \Xi_{s}^{\prime}(x)\right) ; \exists t \in \mathbb{R},\left(t, x, \tau_{0}, 0\right) \in \mathcal{U}\right\}\right)$. Ce sont les projections sur l'espace $T^{*}\left(\mathbb{R}_{x}^{2}\right)$ de $J \cap\left\{\tau=\tau_{0}\right\}$ (resp. $J^{\prime} \cap\left\{\tau=\tau_{0}\right\}$ ).

On remarque qu'au-dessus d'un point $m$ de $S$

$$
\left.T \Lambda_{\tau_{0}}\right|_{m}=T\left(\left\{\xi=0, \tau_{0}+V(x)=0\right\}\right) \oplus \mathbb{R} H,
$$

TOME $130-2002-\mathrm{N}^{\mathrm{O}} 1$ 
où $H(x, 0)=(\nabla V(x) /|\nabla V(x)|,-\nabla V(x))$ : la première projection restreinte à $\Lambda_{\tau_{0}}$ est de rang maximal et $\left.T \Lambda_{\tau_{0}}\right|_{m} ^{\perp}=\left.T \Lambda_{\tau_{0}}\right|_{m}$ pour la forme symplectique $\mathrm{d} \xi \wedge \mathrm{d} x$. De plus, en dehors de $S$, les sous-variétés $\Lambda_{\tau_{0}}$ et $\Lambda_{\tau_{0}}^{\prime}$ sont tissées par le flot hamiltonien de $\tau_{0}+V(x) \pm|\xi|$. On en déduit que $\Lambda_{\tau_{0}}$ (resp. $\left.\Lambda_{\tau_{0}}^{\prime}\right)$ est une sousvariété lagrangienne de $T^{*}\left(\mathbb{R}_{x}^{2}\right)$ admettant un système d'équations locales de la forme $\xi=\nabla_{x} \phi\left(x, \tau_{0}\right)$ (resp. $\xi=\nabla_{x} \phi^{\prime}\left(x, \tau_{0}\right)$ ) où les fonctions $\phi$ et $\phi^{\prime}$ peuvent être choisies de sorte qu'elles dépendent régulièrement du paramètre $\tau_{0}$.

On vérifie aisément que $X_{-s}(x)=X_{s}^{\prime}(x)$ et $\Xi_{-s}(x)=-\Xi_{s}^{\prime}(x)$ car

$$
\begin{aligned}
\frac{\mathrm{d}}{\mathrm{ds}}\left(X_{-s}\right) & =-\dot{X}_{-s}=\operatorname{sgn}(-s) \frac{\Xi_{-s}}{\left|\Xi_{-s}\right|}=\operatorname{sgn}(s) \frac{-\Xi_{-s}}{\left|\Xi_{-s}\right|}, \\
\frac{\mathrm{d}}{\mathrm{ds}}\left(-\Xi_{-s}\right) & =\dot{\Xi}_{-s}=-\nabla V\left(X_{-s}\right) .
\end{aligned}
$$

Dès lors, si $(x, \xi) \in \Lambda_{\tau_{0}}$ alors $(x,-\xi) \in \Lambda_{\tau_{0}}^{\prime}$, et on peut choisir $\phi^{\prime}=-\phi$.

\section{Un résultat général de restitution de l'énergie par le croisement}

Dans ce paragraphe, nous montrons que l'énergie d'une solution ne peut rester localisée sur l'ensemble critique $S$.

Proposition 5. - Si $\mu$ est une mesure semi-classique d'une famille $\left(\psi^{h}\right)$ de solutions du système (3), alors

$$
\mu(\{\xi=0, \nabla V(x) \neq 0\})=0 .
$$

La preuve de la proposition 5 est basée sur le lemme suivant.

LEMME 5. - Soit $\mu$ une mesure semi-classique d'une famille de solutions du système (3), alors

$$
\begin{aligned}
(\tau+V(x)) \partial_{t} \mu-(\tau+V(x)) & \nabla V(x) \cdot \nabla_{\xi} \mu-\xi \cdot \nabla_{x} \mu \\
= & \frac{1}{2}(A(\nabla V(x)) \mu+\mu A(\nabla V(x))) .
\end{aligned}
$$

Preuve du lemme 5. - Considérons l'opérateur $\Gamma$ défini par

$$
\begin{aligned}
\Gamma & =\left(\frac{h}{i} \partial_{t}+V(x)-A\left(h D_{x}\right)\right) \circ\left(\frac{h}{i} \partial_{t}+V(x)+A\left(h D_{x}\right)\right) \\
& =\left(\frac{h}{i} \partial_{t}+V(x)\right)^{2}+h^{2} \Delta+\left[V(x), A\left(h D_{x}\right)\right] .
\end{aligned}
$$

On a $\Gamma \psi^{h}=0$. L'opérateur $\Gamma$ ayant un symbole principal scalaire, on peut utiliser la méthode d'énergie usuelle. Pour tout $a \in \mathcal{C}_{0}^{\infty}\left(\mathbb{R}_{t, x}^{3} \times \mathbb{R}_{\tau, \xi}^{3}\right)$ à valeurs matricielles,

$$
\frac{1}{h}\left(\operatorname{op}_{h}(a) \psi^{h} \mid \Gamma \psi^{h}\right)_{L^{2}\left(\mathbb{R}_{t, x}^{3}\right)}-\frac{1}{h}\left(\operatorname{op}_{h}(a) \Gamma \psi^{h} \mid \psi^{h}\right)_{L^{2}\left(\mathbb{R}_{t, x}^{3}\right)}=0
$$

BULLETIN DE LA SOCIÉTÉ MATHÉMATIQUE DE FRANCE 
Compte tenu de $\Gamma^{*}=\left((h / i) \partial_{t}+V(x)\right)^{2}+h^{2} \Delta-[V(x), A(h D)]$, le calcul pseudodifférentiel usuel donne

$$
\begin{aligned}
\frac{1}{h}\left(\Gamma^{*} \mathrm{op}_{h}(a)-\mathrm{op}_{h}(a) \Gamma\right)=\mathrm{op}_{h} & \left(\frac{1}{i}\left\{(\tau+V(x))^{2}-|\xi|^{2}, a\right\}\right. \\
+ & i\{V(x), A(\xi)\} a+i a\{V(x), A(\xi)\})+o(1)
\end{aligned}
$$

dans $\mathcal{L}\left(L^{2}\left(\mathbb{R}^{d}\right)\right)$. Lorsque $h$ tend vers 0 , on obtient

$$
\begin{array}{r}
\int_{\mathbb{R}_{t, x, \tau, \xi}^{6}} \operatorname{tr}\left(\left(\left\{(\tau+V(x))^{2}-|\xi|^{2}, a(t, x, \tau, \xi)\right\}-\{V(x), A(\xi)\} a(t, x, \tau, \xi)\right.\right. \\
-a(t, x, \tau, \xi)\{V(x), A(\xi)\}) \mu(\mathrm{d} t, \mathrm{~d} x, \mathrm{~d} \tau, \mathrm{d} \xi))=0 .
\end{array}
$$

Cette égalité étant valable pour tout symbole matriciel $a$ - en particulier pour un symbole de la forme $a=\tilde{a} E_{i, j}$ où $\tilde{a}$ est scalaire et $E_{i, j}$ est la matrice $E_{i, j}=\left(\delta_{i, k} \delta_{j, \ell}\right)_{k, \ell}$ - on obtient (32) une fois remarqué que l'on a $\{V(x), A(\xi)\}=-A(\nabla V(x))$.

On utilise alors (32) de la façon suivante. Soit $\Phi \in \mathcal{C}_{0}^{\infty}\left(\mathbb{R}_{\xi}^{2}\right), 0 \leq \Phi \leq 1$, avec $\Phi(\xi)=0$ si $|\xi| \geq 2$ et $\Phi(\xi)=1$ si $|\xi| \leq 1$. Alors pour $\epsilon>0$, l'équation (32) implique

$$
\begin{aligned}
\Phi\left(\frac{\xi}{\epsilon}\right)\left((\tau+V(x)) \partial_{t} \mu-(\right. & \left.+V(x)) \nabla V(x) \cdot \nabla_{\xi} \mu-\xi \cdot \nabla_{x} \mu\right) \\
= & \frac{1}{2} \Phi\left(\frac{\xi}{\epsilon}\right)(A(\nabla V(x)) \mu+\mu A(\nabla V(x))) .
\end{aligned}
$$

En utilisant que $\mu$ est supportée dans l'ensemble caractéristique $\{\tau+V(x) \pm|\xi|=$ $0\}$, on trouve par convergence dominée que le membre de gauche de l'équation (33) tend vers 0 au sens des distributions lorsque $\epsilon$ tend vers 0 , ce qui implique

$$
A(\nabla V(x)) \mathbf{1}_{\xi=0} \mu+\mathbf{1}_{\xi=0} \mu A(\nabla V(x))=0 .
$$

On utilise alors le lemme élémentaire suivant :

Lemme 6. - Soit $\alpha \in \mathbb{R}^{2} \backslash\{0\}$ et $M \in \mathcal{M}_{2}(\mathbb{C})$ une matrice hermitienne positive telle que $A(\alpha) M+M A(\alpha)=0$; alors $M=0$.

Preuve du lemme 6. - Soit $\left(e_{+}, e_{-}\right)$une base orthogonale de vecteurs propres de $A(\alpha)$, alors $A(\alpha) M e_{ \pm}=\mp|\alpha| M e_{ \pm}$. Par définition de $e_{ \pm}$, il existe $\lambda_{+}, \lambda_{-} \in \mathbb{R}$ tels que $M e_{ \pm}=\lambda_{ \pm} e_{\mp}$. Ceci implique $\left\langle M e_{ \pm} \mid e_{ \pm}\right\rangle=0$; la matrice $M$ étant hermitienne positive, on en déduit $M=0$.

Par le théorème de Radon-Nikodym, il existe une fonction $M$ appartenant à $L^{1}\left(\mathbb{R}_{t, x, \tau}^{4} ; \operatorname{tr}\left(\mu \mathbf{1}_{\xi=0}\right)\right)$ et à valeurs dans les matrices hermitiennes positives telle que

$$
\mathbf{1}_{\xi=0} \mu(t, x, \tau, \xi)=M(t, x, \tau) \operatorname{tr}\left(\mu \mathbf{1}_{\xi=0}\right) .
$$

L'équation (34) et le lemme 6 impliquent alors $M=0$ d'où (31). La proposition 5 est démontrée.

TOME $130-2002-\mathrm{N}^{\mathrm{O}} 1$ 


\section{Propagation le long des trajectoires critiques}

Dans ce paragraphe, nous nous intéressons à l'évolution en dehors de $\xi=0$ des mesures à deux échelles associées à une famille $\left(\psi^{h}\right)$ de solutions du système (3) et aux sous-variétés $J$ et $J^{\prime}$. Nous commençons par établir un lemme de séparation des modes qui permet de ramener notre système à l'étude de deux équations scalaires. Nous appliquerons alors les résultats du paragraphe 2.5.

Il suffit de travailler au voisinage d'un point $m_{1}=\left(t_{1}, x_{1}, \tau_{1}, \xi_{1}\right)$ de $\Sigma \cap I \backslash\{\xi=0\}$. Supposons par exemple que $m_{1} \in \Sigma^{+} \cap I$. Soit $\chi \in \mathcal{C}_{0}^{\infty}\left(T^{*} \mathbb{R}^{2}\right)$ valant 1 près de $\left(x_{1}, \xi_{1}\right)$ et telle que, pour tout $(x, \xi)$ dans le support de $\chi$, il existe un repère mobile $\left(e^{+}(\xi), e^{-}(\xi)\right)$ de $\mathbb{R}^{2}$ constitué de vecteurs propres unitaires de $A(\xi)$ dépendant de $\xi$ de façon $\mathcal{C}^{\infty}$.

Soit $\left(u_{+}^{h}\right)$ la famille de fonctions de $L^{2}\left(\mathbb{R}^{2}\right)$ définie par

$$
\left\{\begin{array}{l}
i h \partial_{t} u_{+}^{h}=\left(\left|h D_{x}\right|+V(x)\right) u_{+}^{h}, \\
u_{+}^{h}\left(t_{1}\right)=\left\langle\operatorname{op}_{h}\left(\chi e^{+}\right) \mid \psi^{h}\left(t_{1}\right)\right\rangle
\end{array}\right.
$$

où $\langle\cdot \mid \cdot\rangle$ désigne le produit scalaire sur $\mathbb{C}^{2}$.

Lemme 7. - Il existe un voisinage ouvert $\mathcal{U}$ de $m_{1}$ tel que, pour tout $\Phi$ dans $\mathcal{C}_{0}^{\infty}(\mathcal{U})$,

$$
\mathrm{op}_{h}(\Phi) \psi^{h}=\mathrm{op}_{h}\left(\Phi e^{+}\right) u_{+}^{h}+o(1) \quad \text { dans } \quad L^{2}\left(\mathbb{R}^{3}\right) .
$$

Preuve du lemme \%. - Posons $\tilde{u}_{ \pm}^{h}=\left\langle\operatorname{op}_{h}\left(\chi e^{ \pm}\right) \mid \psi^{h}\right\rangle$. Pour toute fonction $\tilde{\chi}=\tilde{\chi}(x, \xi)$ supportée à l'intérieur de $\{\chi=1\}$, on a

$$
\mathrm{op}_{h}(\tilde{\chi})\left(\psi^{h}-\mathrm{op}_{h}\left(\chi e^{+}\right) \tilde{u}_{+}^{h}-\mathrm{op}_{h}\left(\chi e^{-}\right) \tilde{u}_{-}^{h}\right)=o(1) .
$$

De plus, $\left(\tilde{u}_{ \pm}^{h}\right)$ vérifie

$$
i h \partial_{t} \tilde{u}_{ \pm}^{h}=\left( \pm\left|h D_{x}\right|+V(x)\right) \tilde{u}_{ \pm}^{h}+h f_{ \pm}^{h},
$$

où

$$
f_{ \pm}^{h}=\frac{1}{i} \mathrm{op}_{h}\left(\chi\left\{V, e^{ \pm}\right\}\right) \psi^{h}+\mathrm{op}_{h}\left(\chi^{\prime}\right) \psi^{h}+o(1),
$$

avec $\chi^{\prime}=0$ à l'intérieur de $\{\chi=1\}$.

Puisque $f^{h}$ est bornée dans $L^{2}$, la mesure semi-classique de $\left(\tilde{u}_{ \pm}^{h}\right)$ est supportée dans $\Sigma^{ \pm}$.

Par ailleurs, la base $\left(e^{+}, e^{-}\right)$étant orthonormée, il existe une fonction $\mathcal{C}_{0}^{\infty}$, $\gamma$, telle que, sur le support de $\chi$,

$$
\left\{V, e^{ \pm}\right\}= \pm \gamma e^{\mp}
$$

Il en résulte que

$$
f_{ \pm}^{h}= \pm \frac{1}{i} \mathrm{op}_{h}(\chi \gamma) \tilde{u}_{\mp}^{h}+\mathrm{op}_{h}\left(\chi^{\prime}\right) \psi^{h}+o(1) .
$$

BULletin DE LA SOCiÉtÉ MATHÉmATiQUe DE FRANCE 
Considérons une famille de fonctions $\mathcal{C}_{0}^{\infty}, \tilde{\chi}_{t}=\tilde{\chi}_{t}(x, \xi)$ satisfaisant à

$$
\left\{\tau+V(x)+|\xi|, \tilde{\chi}_{t}\right\}=0
$$

supportée à l'intérieur de $\{\chi=1\}$ et valant 1 près de $m_{1}$. On a, compte-tenu de la localisation des mesures semi-classiques de $\left(\tilde{u}_{-}^{h}\right)$ et de $\left(\tilde{u}_{+}^{h}-u_{+}^{h}\right)$,

$$
\begin{aligned}
& \left\|\operatorname{op}_{h}\left(\tilde{\chi}_{t}\right)\left(\tilde{u}_{+}^{h}-u_{+}^{h}\right)\right\|_{L^{2}\left(\mathbb{R}_{t, x}^{3}\right)}^{2} \\
& \quad=o(1)-2 \operatorname{Re}\left(\int_{t_{1}}^{t}\left(\operatorname{op}_{h}\left(\tilde{\chi}_{s} \gamma\right) \tilde{u}_{-}^{h}(s) \mid \operatorname{op}_{h}\left(\tilde{\chi}_{s}\right)\left(\tilde{u}_{+}^{h}(s)-u_{+}^{h}(s)\right)\right)\right)=o(1) .
\end{aligned}
$$

En revenant à la formule $(37)$ et en choisissant un ouvert $\mathcal{U}$ contenu dans l'intérieur de $\left\{\tilde{\chi}_{t}(x, \xi)=1\right\}$ et ne rencontrant pas $\Sigma^{-}$, on obtient le lemme 7 .

Le lemme 7 a pour corollaire immédiat que les mesures à deux échelles $\nu$ et $\nu^{\prime}$ de la famille $\left(\psi^{h}\right)$ pour $J$ et $J^{\prime}$ respectivement vérifient dans $\mathcal{U}$

$$
\nu=\nu^{+, p} \Pi^{+}, \quad \nu^{\prime}=\nu^{+, f} \Pi^{+},
$$

où les mesures $\nu^{+, p}$ et $\nu^{+, f}$ sont les mesures à deux échelles de la famille $\left(u_{+}^{h}\right)$ pour les sous-variétés $J$ et $J^{\prime}$ respectivement.

On obtient un résultat similaire si $m_{1} \in \Sigma^{-} \cap I$, en introduisant la solution $u_{-}^{h}$ de

$$
\left\{\begin{array}{l}
i h \partial_{t} u_{-}^{h}=\left(-\left|h D_{x}\right|+V(x)\right) u_{-}^{h}, \\
u_{-}^{h}\left(t_{1}\right)=\left\langle\operatorname{op}_{h}\left(\chi e^{-}\right) \mid \psi^{h}\left(t_{1}\right)\right\rangle .
\end{array}\right.
$$

La proposition 2 implique alors le théorème 2 dont nous allons donner une version plus précise dans la suite. Soit $I$ l'hypersurface d'équation $\omega(x, \tau) \wedge \xi=0$. Comme $I \cap \Sigma^{+}=J^{+, p} \cup J^{+, f}$ avec $J^{+, p} \subset J$ et $J^{+, f} \subset J^{\prime}$, le lemme 4 appliqué à la famille $\left(u_{+}^{h}\right)$ permet d'identifier $\nu_{I}$ avec $\nu=\nu^{+, p} \Pi^{+}$ au-dessus de $J^{+, p}$ et avec $\nu^{\prime}=\nu^{+, f} \Pi^{+}$au-dessus de $J^{+, f}$. En utilisant $\left(u_{-}^{h}\right)$, on identifie $\nu_{I}$ avec $\nu^{\prime}=\nu^{-, p} \Pi^{-}$au-dessus de $J^{-, p}$ et avec $\nu=\nu^{-, f} \Pi^{+}$ au-dessus de $J^{-, f}$. Le théorème suivant décrit les équations de transport vérifiées par $\nu_{I}$ et constitue un énoncé équivalent au théorème 2 compte tenu des identifications que nous venons de faire.

THÉORÈme $2^{\prime}$. - On suppose que $\left(\psi^{h}\right)$ est solution de $(3)$. On désigne par $\nu_{I}$ la mesure semi-classique à deux échelles associée à I munie de l'équation $\{\omega(x, \tau) \wedge \xi=0\}$. Alors

$$
\nu_{I}(t, x, \tau, \xi, \eta)=\nu^{+}(t, x, \tau, \xi, \eta) \Pi^{+}(\xi)+\nu^{-}(t, x, \tau, \xi, \eta) \Pi^{-}(\xi),
$$

où $\nu^{ \pm}$sont des mesures positives sur $I \times \overline{\mathbb{R}}$, portées respectivement par $\Sigma^{ \pm} \cap I$ et vérifiant les équations

$$
\partial_{t} \nu^{ \pm} \pm \frac{\xi}{|\xi|} \cdot \nabla_{x} \nu^{ \pm}-\nabla V(x) \cdot \nabla_{\xi} \nu^{ \pm} \pm(\nabla \cdot \omega) \partial_{\eta}\left(\eta \nu^{ \pm}\right)=0
$$

TOME $130-2002-\mathrm{N}^{\mathrm{O}} 1$ 
au-dessus de $J^{ \pm, p} \backslash S$, et

$$
\partial_{t} \nu^{ \pm} \pm \frac{\xi}{|\xi|} \cdot \nabla_{x} \nu^{ \pm}-\nabla V(x) \cdot \nabla_{\xi} \nu^{ \pm} \mp(\nabla \cdot \omega) \partial_{\eta}\left(\eta \nu^{ \pm}\right)=0
$$

au-dessus de $J^{ \pm, f} \backslash S$.

Preuve du théorème $2^{\prime}$. - Les équations (40) et (41) sont une conséquence de la proposition 2 appliquée aux familles de solutions des systèmes (35) et (38) : les mesures $\nu^{ \pm}$vérifient dans $\mathcal{U}$ l'équation

$$
\partial_{t} \nu^{ \pm} \pm \frac{\xi}{|\xi|} \cdot \nabla_{x} \nu^{ \pm}-\nabla V(x) \cdot \nabla_{\xi} \nu^{ \pm}+c^{ \pm} \partial_{\eta}\left(\eta \nu^{ \pm}\right)=0
$$

où $c^{ \pm}=c^{ \pm}(x, \tau, \xi)$ est tel que $\{\tau \pm|\xi|+V(x), \omega(x, \tau) \wedge \xi\}=c^{ \pm}(x, \tau, \xi) \omega(x, \tau) \wedge \xi+\tilde{c}^{ \pm}(x, \tau, \xi)(\tau \pm|\xi|+V(x))$.

Il reste à calculer $c^{ \pm}$. Remarquons que

$$
\{\tau \pm|\xi|+V(x), \omega(x, \tau) \wedge \xi\}=\nabla V(x) \wedge \omega(x, \tau) \pm \frac{\xi}{|\xi|} \cdot \nabla_{x}(\xi \wedge \omega(x, \tau)) .
$$

D'après (29), le vecteur $(\tau+V(x)) \omega(x, \tau)$ est un gradient en $x$ donc

$$
\nabla_{x} \wedge((\tau+V(x)) \omega(x, \tau))=0,
$$

ce qui implique

$$
\nabla V(x) \wedge \omega(x, \tau)=-(\tau+V(x)) \nabla_{x} \wedge \omega(x, \tau) .
$$

D'autre part,

$$
\xi \cdot \nabla_{x}(\omega \wedge \xi)=-(\xi \cdot \omega)^{2} \nabla_{x} \wedge \omega-(\xi \cdot \omega)(\omega \wedge \xi) \nabla_{x} \cdot \omega .
$$

Les relations (42) et (43) donnent sur $\Sigma=\{\tau+V(x)= \pm|\xi|\}$

$$
c^{ \pm}(x, \xi, \tau) \omega \wedge \xi= \pm \frac{(\xi \wedge \omega)^{2}}{|\xi|} \nabla_{x} \wedge \omega \mp(\omega \wedge \xi) \frac{(\xi \cdot \omega)}{|\xi|} \nabla_{x} \cdot \omega .
$$

On en déduit que sur $\Sigma \cap I$, on a

$$
c^{ \pm}(x, \xi, \tau)=\mp \frac{\xi \cdot \omega}{|\xi|} \nabla_{x} \cdot \omega .
$$

Or, d'après la proposition 4,

$$
\text { si } \xi \in J, \quad \xi=(\tau+V) \omega \text { et si } \xi \in J^{\prime}, \quad \xi=-(\tau+V) \omega,
$$

avec $\tau+V=\mp|\xi|$, ce qui achève la preuve du théorème $2^{\prime}$ 


\section{Réduction à un problème modèle}

6.1. Transformation canonique. - Nous allons redresser l'hypersurface $I$ grâce à la transformation canonique suivante.

Proposition 6. - Il existe une transformation canonique locale $\kappa$ sur $T^{*} \mathbb{R}^{3}$,

$$
\kappa(t, x, \tau, \xi)=(s, z, \sigma, \zeta),
$$

et des fonctions $\mathcal{C}^{\infty}, \lambda$ et $\mu$, strictement positives près de $\left(x_{0}, \tau_{0}\right)$ telles que

$$
\left\{\begin{aligned}
\sigma & =-\lambda(x, \tau)(\tau+V(x)), \\
s & =\lambda(x, \tau) \omega(x, \tau) \cdot \xi, \\
\zeta_{2} & =\lambda(x, \tau) \mu(x, \tau) \omega(x, \tau) \wedge \xi .
\end{aligned}\right.
$$

De plus, on peut choisir $z=z(x, \tau)$ de sorte que $(x, \tau) \mapsto(z, \sigma)$ soit un difféomorphisme local.

Preuve de la proposition 6. - En utilisant le théorème de Darboux (cf. par exemple [16]), il suffit de montrer qu'il existe $\lambda=\lambda(x, \tau), \mu=\mu(x, \tau)$, strictement positives telles que si $s, \sigma$ et $\zeta_{2}$ sont définis par (44), on ait

$$
\{\sigma, s\}=1, \quad\left\{\zeta_{2}, s\right\}=0, \quad\left\{\sigma, \zeta_{2}\right\}=0 .
$$

Le lemme suivant nous permet de construire $\lambda$.

LEMme 8. - Soit $W=W\left(x_{1}, x_{2}\right), \mathcal{C}^{\infty}$ avec $\mathrm{d} W \neq 0$, supposons qu'il existe une fonction $\mathcal{C}^{\infty}$ g telle que

$$
\left\{\begin{array}{l}
\mathrm{d} g=0 \text { si } W=0, \\
\text { Hess } g \neq 0 \text { si } W=0 .
\end{array}\right.
$$

Alors, au voisinage de tout point où $W$ s'annule, il existe une fonction $\lambda, \mathcal{C}^{\infty}$ strictement positive telle que

$$
g=\mathrm{Cte} \pm \frac{1}{2}(\lambda W)^{2} .
$$

Preuve du lemme 8. - Le problème est invariant par changement de coordonnées dans $\mathbb{R}^{2}$; on peut donc supposer $W(x)=x_{1}$ et se placer au voisinage de 0 . La fonction $g$ vérifie dans ces nouvelles coordonnées

$$
\forall x_{2} \in \mathbb{R}, \quad \mathrm{d} g\left(0, x_{2}\right)=0 \text { et } \mathrm{d}^{2} g\left(0, x_{2}\right) \neq 0 .
$$

Nécessairement, $g\left(0, x_{2}\right)=$ Cte; notons a cette constante. D'après la formule de Taylor, il existe une fonction $\mathcal{C}^{\infty} \gamma$ telle que

$$
g(x)=a+\frac{x_{1}^{2}}{2} \gamma(x)
$$

Comme $\mathrm{d}^{2} g\left(0, x_{2}\right)=\left(\begin{array}{cc}\gamma\left(0, x_{2}\right) & 0 \\ 0 & 0\end{array}\right) \neq 0$, on en déduit que $\gamma(x) \neq 0$ dans un voisinage de 0 ; la fonction $\lambda=\sqrt{|\gamma|}$ est alors $\mathcal{C}^{\infty}$ et résout notre problème.

TOME $130-2002-\mathrm{N}^{\mathrm{O}} 1$ 
On sait par la proposition 4 qu'il existe une fonction $\phi$ vérifiant $(29), \phi$ satisfait donc aux hypothèses du lemme précédent avec $W_{\tau}(x)=V(x)+\tau$. Quitte à changer $\phi$ en $-\phi$ et à la modifier par addition d'une fonction de $\tau$ si nécessaire, il existe une fonction $\lambda=\lambda(x, \tau)$ strictement positive, $\mathcal{C}^{\infty}$ dans les variable $x$ et $\tau$, telle que

$$
\phi(x, \tau)=\frac{1}{2}(\lambda(x, \tau)(V(x)+\tau))^{2} .
$$

C'est cette fonction $\lambda$ que nous allons utiliser. Du fait que $|\nabla \phi|=|V(x)+\tau|$, la fonction $\lambda$ vérifie certaines propriétés qui nous seront utiles dans la suite et que nous allons rassembler dans la remarque suivante.

Remarque. - Comme $\nabla \phi=\lambda(\tau+V) \nabla_{x}(\lambda(\tau+V))=(\tau+V) \omega$, on a

$$
\lambda \nabla_{x}(\lambda(\tau+V))=\omega .
$$

De plus $1=\left|\lambda \nabla_{x}(\lambda(\tau+V))\right|=\lambda^{2}|\nabla V|$ sur $\{\tau+V(x)=0\}$, donc

$$
(\lambda(x, \tau))^{2}=\frac{1}{|\nabla V(x)|} \quad \text { sur } \quad\{\tau+V(x)=0\} .
$$

Revenons à la démonstration de la proposition 6 . Considérons

$$
\sigma=-\lambda(x, \tau)(\tau+V(x)) \text { et } \quad s=\lambda(x, \tau) \omega(x, \tau) \cdot \xi .
$$

Le calcul donne

$$
\{\sigma, s\}=\lambda(x, \tau) \omega(x, \tau) \cdot \nabla_{x}(\lambda(x, \tau)(V(x)+\tau))=1
$$

et, pour toute fonction $\mu=\mu(x, \tau)$, par (45),

$$
\begin{aligned}
& \{\sigma, \lambda(x, \tau) \mu(x, \tau) \omega(x, \tau) \wedge \xi\} \\
& \quad=\lambda(x, \tau) \mu(x, \tau) \omega(x, \tau) \wedge \nabla_{x}(\lambda(x, \tau)(V(x)+\tau))=0 .
\end{aligned}
$$

Recherchons alors $\mu$ telle que $\zeta_{2}=\lambda(x, \tau) \mu(x, \tau) \omega(x, \tau) \wedge \xi$ vérifie $\left\{s, \zeta_{2}\right\}=0$. Compte tenu de

$$
\{\lambda \omega \cdot \xi, \lambda \mu \omega \wedge \xi\}=\left[\lambda \omega, \lambda \mu \omega^{\perp}\right] \cdot \xi,
$$

le lemme suivant nous permet de conclure.

Lemme 9. - Soient $X_{1}$ et $X_{2}$ deux champs de vecteurs de $\mathbb{R}^{2}$ partout non nuls et tels que $X_{1} \cdot X_{2}=0$. Alors

$$
\left[X_{1}, X_{2}\right]=0 \Longleftrightarrow \nabla \wedge\left(\frac{X_{1}}{\left|X_{1}\right|^{2}}\right)=0 \text { et } \nabla \wedge\left(\frac{X_{2}}{\left|X_{2}\right|^{2}}\right)=0 .
$$

Appliqué à $X_{1}=\lambda \omega$ et $X_{2}=\lambda \mu \omega^{\perp}$, le lemme 9 établit l'existence de $\kappa$ sous les conditions

$$
\nabla \wedge\left(\frac{\omega}{\lambda}\right)=0 \quad \text { et } \quad \nabla \wedge\left(\frac{\omega^{\perp}}{\lambda \mu}\right)=0 .
$$

Comme $\omega / \lambda=\nabla_{x}(\lambda(V(x)+\tau))$ d'après (45), la première condition est vérifiée. Il reste à choisir localement $\mu>0$ telle que $\nabla_{x} \cdot(\omega / \lambda \mu)=0$, ce qui ne présente 
pas de difficulté puisque $\omega / \lambda$ est un champ de vecteur régulier ne s'annulant pas. On choisit alors $z_{1}=\tau$ et $z_{2}=z_{2}(x, \tau)$ tel que

$$
\nabla_{x} z_{2}=\frac{\omega^{\perp}}{\lambda \mu}
$$

de sorte que $(x, \tau) \mapsto(z, \sigma)$ est bien de rang maximal.

Preuve du lemme 9. - Il suffit de raisonner localement près d'un point $x_{0}$. Le fait que $X_{1}$ et $X_{2}$ commutent équivaut à l'existence d'une application $F=F\left(t_{1}, t_{2}\right)$ d'un voisinage de 0 dans $\mathbb{R}^{2}$ sur un voisinage de $x_{0}$ telle que

$$
\partial_{t_{j}} F=X_{j}(F), \quad j=1,2 .
$$

L'application $F$ est un difféomorphisme local; notons $F^{-1}=\left(u_{1}, u_{2}\right)$. En dérivant par rapport à $t_{1}, t_{2}$ les identités

$$
u_{j}\left(F\left(t_{1}, t_{2}\right)\right)=t_{j}
$$

on obtient

$$
\nabla u_{j}(F) \cdot \partial_{t_{k}} F=\delta_{j k},
$$

soit, en utilisant l'orthogonalité de $X_{1}, X_{2}$,

$$
\forall j=1,2, \quad \nabla u_{j}=\frac{X_{j}}{\left|X_{j}\right|^{2}} .
$$

Il en résulte que $\nabla \wedge\left(X_{j} /\left|X_{j}\right|^{2}\right)=0$. Réciproquement, les identités (50) assurent que $\left(u_{1}, u_{2}\right)$ est un difféomorphisme local, dont l'inverse $F$ vérifie (49), donc (48).

6.2. Le système modèle. - Utilisons la transformation canonique précédente pour ramener le système (3) à un système modèle. Commençons par effectuer un changement de fonctions inconnues. Soit $\omega=\omega(x, \tau)$ la fonction définie par la formule (30), et soit $\theta$ la fonction à valeurs réelles définie par

$$
(\cos (2 \theta(x, \tau)),-\sin (2 \theta(x, \tau)))=\omega(x, \tau) .
$$

Soit $R_{\theta}$ la matrice de rotation d'angle $\theta$,

$$
R_{\theta}=\left(\begin{array}{cc}
\cos \theta & -\sin \theta \\
\sin \theta & \cos \theta
\end{array}\right) \text {. }
$$

Remarquons que

$$
R_{\theta} A(\xi) R_{\theta}^{-1}=\left(\begin{array}{cc}
\omega \cdot \xi & \omega \wedge \xi \\
\omega \wedge \xi-\omega \cdot \xi
\end{array}\right)
$$

donc, en notant $R=\mathrm{op}_{h}\left(R_{\theta(x, \tau)}\right)$, on a l'identité

$$
R \mathrm{op}_{h}(\tau+V(x)+A(\xi)) R^{-1}=\mathrm{op}_{h}(\tilde{Q}(x, \tau, \xi))
$$

TOME $130-2002-\mathrm{N}^{\mathrm{O}} 1$ 
où, près de $m_{0}$,

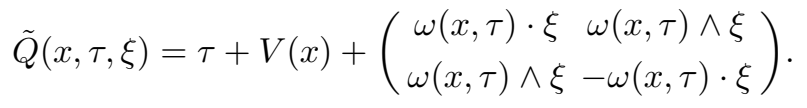

Soit $\nu_{I}$ la mesure à deux échelles associée à $\left(\psi^{h}\right)$ et à $I=\{\omega(x, \tau) \wedge \xi=0\}$. La mesure $\tilde{\nu}=R_{\theta(x, \tau)} \nu_{I} R_{\theta(x, \tau)}^{-1}$ est la mesure à deux échelles pour l'hypersurface $I=\{\omega(x, \tau) \wedge \xi=0\}$ de la famille $\left(R \psi^{h}\right)$ qui satisfait à

$$
\mathrm{op}_{h}(\tilde{Q}) R \psi^{h}=0 \text {. }
$$

D'après (51) et (7),

- $\operatorname{si}(t, x, \tau, \xi) \in J^{-, p} \cup J^{+, p}$,

$$
\begin{aligned}
& R_{\theta(x, \tau)} \Pi^{+}(\xi) R_{\theta(x, \tau)}^{-1}=\left(\begin{array}{ll}
0 & 0 \\
0 & 1
\end{array}\right)=E_{2}, \\
& R_{\theta(x, \tau)} \Pi^{-}(\xi) R_{\theta(x, \tau)}^{-1}=\left(\begin{array}{ll}
1 & 0 \\
0 & 0
\end{array}\right)=E_{1},
\end{aligned}
$$

- $\operatorname{si}(t, x, \tau, \xi) \in J^{-, f} \cup J^{+, f}$,

$$
\begin{aligned}
& R_{\theta(x, \tau)} \Pi^{+}(\xi) R_{\theta(x, \tau)}^{-1}=E_{1}, \\
& R_{\theta(x, \tau)} \Pi^{-}(\xi) R_{\theta(x, \tau)}^{-1}=E_{2} .
\end{aligned}
$$

La formule (39) s'écrit alors

$$
\tilde{\nu}= \begin{cases}\nu^{+} E_{2}+\nu^{-} E_{1} & \text { au-dessus de } J^{-, p} \cup J^{+, p}, \\ \nu^{+} E_{1}+\nu^{-} E_{2} & \text { au-dessus de } J^{-, f} \cup J^{+, f} .\end{cases}
$$

Soit $m_{0}=\left(s=0, z_{0}, \sigma=0, \zeta_{1,0}, \zeta_{2}=0\right) \in T^{*}\left(\mathbb{R}^{3}\right)$, et soit $\kappa$ une transformation canonique définie comme au $\S 3.2$, au voisinage de $m_{0}$. Soit $U$ un opérateur intégral de Fourier unitaire associé à la transformation canonique $\kappa$ et

$$
v^{h}=U \operatorname{op}_{h}\left(\lambda(x, \tau)^{-1 / 2}\right) R \psi^{h} .
$$

Soit $Q$ la matrice définie par

$$
Q=\left(\begin{array}{cc}
-\sigma+s & \alpha \zeta_{2} \\
\alpha \zeta_{2} & -\sigma-s
\end{array}\right)
$$

avec $\alpha=1 / \mu$. En utilisant (20), on obtient

$$
\begin{aligned}
\operatorname{op}_{h}(Q) v^{h} & =\operatorname{op}_{h}(Q) U \mathrm{op}_{h}\left(\lambda(x, \tau)^{-1 / 2}\right) R \psi_{h} \\
& =U \operatorname{op}_{h}(Q \circ \kappa) \operatorname{op}_{h}\left(\lambda(x, \tau)^{-1 / 2}\right) R \psi_{h}+O\left(h^{2}\right) \\
& =U \operatorname{op}_{h}(\lambda(x, \tau) \tilde{Q}(x, \tau, \xi)) \operatorname{op}_{h}\left(\lambda(x, \tau)^{-1 / 2}\right) R \psi_{h} .
\end{aligned}
$$

Mais par le calcul de Weyl, on obtient $\mathrm{op}_{h}(\lambda(x, \tau) \tilde{Q}(x, \tau, \xi)) \mathrm{op}_{h}\left(\lambda(x, \tau)^{-1 / 2}\right)=\operatorname{op}_{h}\left(\lambda(x, \tau)^{1 / 2}\right) \mathrm{op}_{h}(\tilde{Q}(x, \tau, \xi))+O\left(h^{2}\right)$. 
On en déduit que la famille $\left(v^{h}\right)$ est localement solution du système (14), au sens où, pour toute fonction $\beta \in \mathcal{C}_{0}^{\infty}$ à support dans un voisinage $U$ assez petit de $m_{0}$,

$$
\mathrm{op}_{h}(\beta) \operatorname{op}_{h}(Q) v^{h}=O\left(h^{2}\right) .
$$

D'après la proposition 1 , la mesure $\underline{\nu}=\tilde{\nu} \circ \bar{N}(\kappa)^{-1}$ est la mesure à deux échelles de la famille $\left(v^{h}\right)$ pour l'équation $\zeta_{2} / \lambda \mu=0$ de $I$. Les résultats des sections précédentes assurent que $\underline{\nu}$ ne charge pas l'ensemble singulier $S=\left\{\sigma=s=\zeta_{2}=0\right\} \subset I$. Notons $\underline{\nu}^{ \pm}=\bar{\nu}^{ \pm} \circ \bar{N}(\kappa)^{-1}$. Les mesures scalaires $\underline{\nu}^{ \pm}$ sont portées respectivement par $\left\{\zeta_{2}=0,-\sigma \pm|s|=0\right\}$ et vérifient les équations de transport suivantes :

$$
\left\{\begin{array}{l}
\partial_{s} \underline{\nu}^{ \pm} \pm \partial_{\sigma} \underline{\nu}^{ \pm}=0 \text { dans }\{s>0\}, \\
\partial_{s} \underline{\nu}^{ \pm} \mp \partial_{\sigma} \underline{\nu}^{ \pm}=0 \text { dans }\{s<0\} .
\end{array}\right.
$$

\section{Analyse à l'infini}

Dans ce paragraphe, on étudie la propagation des mesures à deux échelles à l'infini sur le fibré normal à $I$, pour le problème modèle (53) obtenu au paragraphe 6.2 précédent. On note

$$
A(s, z, \sigma, \zeta)=\left(\begin{array}{cc}
s & \alpha(\sigma, z) \zeta_{2} \\
\alpha(\sigma, z) \zeta_{2} & -s
\end{array}\right),
$$

de sorte que $Q=-\sigma+A$.

Notre objectif dans ce paragraphe est de démontrer les identités suivantes :

$$
\left.\mathbf{1}_{|\eta|=+\infty} \underline{\nu}^{ \pm}\right|_{s=0^{+}}=\left.\mathbf{1}_{|\eta|=+\infty} \underline{\nu}^{ \pm}\right|_{s=0^{-}} .
$$

Pour ce faire, nous démontrons tout d'abord une estimation a priori sur les solutions d'un système du type (53). Notons au préalable que si l'on fixe $\chi$ dans $\mathcal{C}_{0}^{\infty}(U)$ (valant 1 près de $m_{0}$ ), l'équation (53) et le calcul symbolique entraînent que $\underline{v}^{h}=\operatorname{op}_{h}(\chi) v^{h}$ vérifie

$$
\left\|\operatorname{op}_{h}(Q) \underline{v}^{h}\right\|_{L^{2}}=O(h) .
$$

De plus, on peut supposer que la fonction $\alpha$ est à support compact sur $\mathbb{R}_{\sigma, z}^{3}$, quitte à la tronquer loin de $U$.

\subsection{Estimation d'énergie hyperbolique}

Proposition 7. - Soit $\left(\underline{v}^{h}\right)$ une famille bornée de $L^{2}\left(\mathbb{R}_{s} \times \mathbb{R}_{z}^{2}, \mathbb{C}^{2}\right)$ solution du système $(55)$, où $Q$ est donnée par $(52)$. Soit $\rho \in \mathcal{C}_{0}^{\infty}(\mathbb{R})$ une fonction valant 1 près de 0 . Alors il existe $\epsilon>0$ et $h_{0}>0$ tels que la famille $\left(\rho\left(h D_{z_{2}} / \epsilon\right) \underline{v}^{h}\right)_{h<h_{0}}$ soit bornée dans $L^{\infty}\left(\mathbb{R}_{s}, L^{2}\left(\mathbb{R}_{z}^{2}\right)\right)$.

TOME $130-2002-\mathrm{N}^{\mathrm{O}} 1$ 
Preuve de la proposition \%. - Soit $\tilde{\rho} \in \mathcal{C}_{0}^{\infty}(\mathbb{R})$ à valeurs réelles, valant 1 dans un voisinage du support de $\rho$. Notons

$$
A_{\epsilon}(s, \sigma, z, \zeta)=\left(\begin{array}{cc}
s & \alpha(\sigma, z) \zeta_{2} \tilde{\rho}\left(\zeta_{2} / \epsilon\right) \\
\alpha(\sigma, z) \zeta_{2} \tilde{\rho}\left(\zeta_{2} / \epsilon\right) & -s
\end{array}\right) .
$$

Alors le calcul symbolique pseudodifférentiel assure que $v_{\epsilon}^{h}=\rho\left(h D_{z_{2}} / \epsilon\right) \underline{v}^{h}$ satisfait au système

$$
\left\|i h \partial_{s} v_{\epsilon}^{h}+\mathrm{op}_{h}\left(A_{\epsilon}\right) v_{\epsilon}^{h}\right\|_{L^{2}\left(\mathbb{R}_{s, z}^{3}\right)}=O_{\epsilon}(h)
$$

où $O_{\epsilon}(h)$ désigne une quantité bornée par $C_{\epsilon} h, C_{\epsilon}$ étant une constante dépendant de $\epsilon$. Pour tout $s_{0} \in \mathbb{R}$, écrivons

$$
\begin{aligned}
\left\|v_{\epsilon}^{h}\left(s_{0}\right)\right\|_{L_{z}^{2}}^{2} & =2 \operatorname{Re}\left(\int_{-\infty}^{s_{0}}\left(\partial_{s} v_{\epsilon}^{h}(s) \mid v_{\epsilon}^{h}(s)\right)_{L_{z}^{2}} \mathrm{~d} s\right) \\
& =O_{\epsilon}(1)-\frac{2}{h} \operatorname{Im}\left(\int_{-\infty}^{s_{0}}\left(\left[\operatorname{op}_{h}\left(A_{\epsilon}\right) v_{\epsilon}^{h}\right](s) \mid v_{\epsilon}^{h}(s)\right)_{L_{z}^{2}} \mathrm{~d} s\right)
\end{aligned}
$$

Notons $L=\left(\begin{array}{ll}0 & 1 \\ 1 & 0\end{array}\right)$,

$$
\begin{aligned}
& a(t, z)=\frac{1}{2 \pi} \int_{-\infty}^{+\infty} \mathrm{e}^{i t \sigma} \alpha(\sigma, z) \mathrm{d} \sigma \\
& M_{\epsilon}^{h}(t)=\operatorname{op}_{h}\left(a(t, z) \zeta_{2} \tilde{\rho}\left(\frac{\zeta_{2}}{\epsilon}\right)\right) L \in \mathcal{L}\left(L^{2}\left(\mathbb{R}_{z}^{2}, \mathbb{C}^{2}\right)\right) .
\end{aligned}
$$

Alors, puisque $M_{\epsilon}^{h}(t)^{*}=M_{\epsilon}^{h}(-t)$,

$$
\begin{aligned}
& 2 \operatorname{Im}\left(\left[\operatorname{op}_{h}\left(A_{\epsilon}\right) v_{\epsilon}^{h}\right](s) \mid v_{\epsilon}^{h}(s)\right)_{L_{z}^{2}} \\
& \quad=2 \operatorname{Im}\left(\int_{-\infty}^{+\infty}\left(M_{\epsilon}^{h}(t) v_{\epsilon}^{h}(s-h t), v_{\epsilon}^{h}(s)\right)_{L_{z}^{2}} \mathrm{~d} t\right) \\
& \quad=\frac{1}{i} \int_{-\infty}^{+\infty}\left[\left(M_{\epsilon}^{h}(t) v_{\epsilon}^{h}(s-h t) \mid v_{\epsilon}^{h}(s)\right)_{L_{z}^{2}}-\left(M_{\epsilon}^{h}(-t) v_{\epsilon}^{h}(s) \mid v_{\epsilon}^{h}(s-h t)\right)_{L_{z}^{2}}\right] \mathrm{d} t
\end{aligned}
$$

Retournant à (56), le changement de variables $(t, s) \mapsto(-t, s-h t)$ dans la seconde intégrale donne,

$$
\left\|v_{\epsilon}^{h}\left(s_{0}\right)\right\|_{L_{z}^{2}}=O_{\epsilon}(1)+\frac{1}{i h} \int_{-\infty}^{+\infty}\left(\int_{s_{0}}^{s_{0}+h t}\left(M_{\epsilon}^{h}(t) v_{\epsilon}^{h}(s-h t) \mid v_{\epsilon}^{h}(s)\right)_{L_{z}^{2}} \mathrm{~d} s\right) \mathrm{d} t .
$$

D'après l'inégalité de Gårding (cf. par exemple [6]),

$$
\left\|M_{\epsilon}^{h}(t)\right\|_{\mathcal{L}\left(L_{z}^{2}\right)} \leq \sup _{z, \zeta_{2}}\left|a(t, z) \zeta_{2} \tilde{\rho}\left(\frac{\zeta_{2}}{\epsilon}\right)\right|+h C_{\epsilon} \sup _{\substack{z \\|\alpha| \leq N}}\left|\partial_{z}^{\alpha} a(t, z)\right|
$$

BULLETIN DE LA SOCIÉtÉ MATHÉMATIQUE DE FRANCE 
d'où, en utilisant la décroissance rapide en $t$ de $\sup \left|\partial_{z}^{\alpha} a(t, z)\right|$,

$$
\left\|v_{\epsilon}^{h}\right\|_{L^{\infty}\left(\mathbb{R}, L^{2}\right)}^{2} \leq O_{\epsilon}(1)+\left(C \epsilon+O_{\epsilon}(h)\right)\left\|v_{\epsilon}^{h}\right\|_{L^{\infty}\left(\mathbb{R}, L^{2}\right)}^{2},
$$

ce qui achève la démonstration.

7.2. Réflexion totale. - Une conséquence importante de l'estimation précédente est le lemme suivant.

Lemme 10. - Il existe un voisinage ouvert $U^{\prime} \subset U$ de $m_{0}$ tel que, pour toute fonction $\chi \in \mathcal{C}_{0}^{\infty}\left(U^{\prime}\right)$, la famille $\underline{v}^{h}=\mathrm{op}_{h}(\chi) v^{h}$ vérifie, pour $h$ assez petit, et pour tout compact $K$ de $\mathbb{R}^{6}$, l'estimation

$$
\left|\left(\operatorname{op}_{h}(b) \underline{v}^{h} \mid \underline{v}^{h}\right)\right| \leq C \int_{-\infty}^{+\infty} \sup _{k+|\beta| \leq N} \sup _{(z, \sigma, \zeta) \in \mathbb{R}^{5}}\left|\partial_{\sigma}^{k} \partial_{z}^{\beta} b(s, z, \sigma, \zeta)\right| \mathrm{d} s,
$$

pour tout $b \in \mathcal{C}_{0}^{\infty}(K), N$ étant un entier fixe.

Preuve du lemme 10. - Il suffit d'écrire

$$
\begin{aligned}
& \left(\mathrm{op}_{h}(b) \underline{v}^{h}, \underline{v}^{h}\right) \\
& \left.\quad=\frac{1}{h^{3}} \int_{\mathbb{R}^{3}} k\left(\frac{s+s^{\prime}}{2}, \frac{\zeta-\zeta^{\prime}}{h}, \frac{s-s^{\prime}}{h}, \frac{\zeta+\zeta^{\prime}}{2}\right){\underline{v^{h}}}^{\left(s^{\prime}\right.}, \zeta^{\prime}\right) \overline{\underline{v}^{h}}(s, \zeta) \mathrm{d} s \mathrm{~d} \zeta \mathrm{d} s^{\prime} \mathrm{d} \zeta^{\prime}
\end{aligned}
$$

où $\hat{v}$ désigne la $h$-transformée de Fourier de $v$ par rapport à la variable $z$, et où

$$
k(s, \dot{\zeta}, \dot{s}, \zeta)=\int_{\mathbb{R}^{3}} b(s, z, \sigma, \zeta) \mathrm{e}^{i(\sigma \dot{s}-z \cdot \dot{\zeta})} \frac{\mathrm{d} \sigma \mathrm{d} z}{(2 \pi)^{3}} .
$$

La proposition 7 et le lemme de Schur entraînent alors

$$
\left|\left(\operatorname{op}_{h}(b) \underline{v}^{h} \mid \underline{v}^{h}\right)\right| \leq C \int_{\mathbb{R}^{4}} \sup _{\zeta}|k(s, \dot{\zeta}, \dot{s}, \zeta)| \mathrm{d} s \mathrm{~d} \dot{s} \mathrm{~d} \dot{\zeta},
$$

et l'estimation annoncée s'en déduit, puisque $b \in \mathcal{C}_{0}^{\infty}(K)$.

Venons-en à la démonstration de la formule (54). Les valeurs propres de $A$ et leurs projecteurs associés sont

$$
\begin{aligned}
\lambda\left(s, z, \sigma, \zeta_{2}\right) & =\sqrt{s^{2}+\alpha(\sigma, z)^{2} \zeta_{2}^{2}} \\
\Pi^{ \pm}(s, z, \sigma, \zeta)=\frac{1}{2}\left(1 \pm \frac{A}{\lambda}\right) & =\frac{1}{2}\left(1 \pm \frac{1}{\sqrt{s^{2}+\alpha^{2} \zeta_{2}^{2}}}\left(\begin{array}{cc}
s & \alpha \zeta_{2} \\
\alpha \zeta_{2} & -s
\end{array}\right)\right) .
\end{aligned}
$$

Remarquons que, sur $\zeta_{2}=0$,

$$
\Pi_{\mid s=0^{+}}^{+}=\Pi_{\mid s=0^{-}}^{-}=E_{1}, \quad \Pi_{\mid s=0^{+}}^{-}=\Pi_{\mid s=0^{-}}^{+}=E_{2} .
$$

Rappelons également que

$$
\underline{\nu}^{ \pm}=\operatorname{tr}\left(\underline{\nu} \Pi^{ \pm}\right) .
$$

Nous traitons le cas de $\underline{\nu}^{+}$, celui de $\underline{\nu}^{-}$lui est semblable. La proposition 7 assure que $\underline{\nu}^{+}$est décrite près de $m_{0}$ par une fonction $L^{\infty}$ de $s$ à valeurs mesures

TOME $130-2002-\mathrm{N}^{\mathrm{O}} 1$ 
dans les autres variables; en particulier, $\nu^{+}$ne charge pas $\{s=0\}$. Il en résulte que, pour toute fonction test $a_{0}=a_{0}(z, \sigma, \zeta, \eta)$,

$$
\left\langle\underline{\nu}^{+}\left(s=0^{+}\right)-\underline{\nu}^{+}\left(s=0^{-}\right), a_{0}\right\rangle=\lim _{\delta \rightarrow 0}\left\langle\partial_{s} \underline{\nu}^{+}, a_{0} \rho\left(\frac{s}{\delta}\right)\right\rangle,
$$

où $\rho \in \mathcal{C}_{0}^{\infty}(\mathbb{R})$ vaut 1 près de l'origine. Soit alors $a=a(s, z, \sigma, \zeta, \eta)$ un élément de $\mathcal{A}$ de la forme

$$
a(s, z, \sigma, \zeta, \eta)=a_{0}(z, \sigma, \zeta) \rho\left(\frac{s}{\delta}\right) \rho\left(\frac{\zeta_{2}}{\varepsilon}\right)(1-\rho)\left(\frac{\eta}{R}\right),
$$

où $\varepsilon, \delta$ et le support de $a_{0}$ sont supposés assez petits pour que $a(., \eta) \in \mathcal{C}_{0}^{\infty}\left(U^{\prime}\right)$ pour tout $\eta \in \mathbb{R}$. Par définition de $\underline{\nu}^{+}$,

$$
\left\langle\partial_{s} \underline{\nu}^{+}, a_{0} \rho\left(\frac{s}{\delta}\right)\right\rangle=\lim _{\varepsilon \rightarrow 0} \lim _{R \rightarrow \infty} \lim _{h \rightarrow 0} L_{\delta, \varepsilon, R}^{h}
$$

avec

$$
L_{\delta, \varepsilon, R}^{h}=-\int \partial_{s} a\left(s, z, \sigma, \zeta, \frac{\zeta_{2}}{\sqrt{h}}\right) \operatorname{tr}\left(W^{h} v^{h}(s, z, \sigma, \zeta) \Pi^{+}(s, z, \sigma, \zeta)\right) \mathrm{d} s \mathrm{~d} \sigma \mathrm{d} z \mathrm{~d} \zeta .
$$

En effet, bien que $\Pi^{+}$soit singulière près de $s=\zeta_{2}=0$, l'annulation de $\partial_{s} a$ près de $s=0$ assure que l'on intègre bien $W^{h} v^{h}$ contre une fonction du type $q\left(s, z, \sigma, \zeta, \zeta_{2} / \sqrt{h}\right)$, où $q$ est un élément de $\mathcal{A}$ à valeurs matricielles.

Pour étudier $L_{\delta, \varepsilon, R}^{h}$, nous aurons recours à des techniques d'énergie pseudodifférentielles, et introduisons donc quelques notations à cet effet. Si $q=q(s, z, \sigma, \zeta, \eta)$, nous noterons $\mathrm{op}_{h}^{(2)}(q)$ l'opérateur $\mathrm{op}_{h}\left(\tilde{q}_{h}\right)$, avec

$$
\tilde{q}_{h}(s, z, \sigma, \zeta)=q\left(s, z, \sigma, \zeta, \frac{\zeta_{2}}{\sqrt{h}}\right) .
$$

Il sera parfois utile de revenir à la quantification non semi-classique $(h=1)$, et nous noterons alors

$$
q_{h}^{\sharp}(s, z, \sigma, \zeta)=\tilde{q}_{h}(s, z, h \sigma, h \zeta)=q\left(s, z, h \sigma, h \zeta, \sqrt{h} \zeta_{2}\right),
$$

de sorte que

$$
\mathrm{op}_{h}^{(2)}(q)=\mathrm{op}_{h}\left(\tilde{q}_{h}\right)=\mathrm{op}_{1}\left(q_{h}^{\sharp}\right) .
$$

Passons à l'expression de $L_{\delta, \varepsilon, R}^{h}$, que nous noterons $L^{h}$ pour simplifier. La localisation de $\zeta_{2}$ dans la zone $\left|\zeta_{2}\right| \geq R \sqrt{h}$ assurant que $\Pi^{+}$est une fonction $\mathcal{C}^{\infty}$ à $h$ fixé près du support de $\tilde{a}_{h}$, on a

$$
\begin{aligned}
L^{h}=-\left(\operatorname{op}_{h}^{(2)}\left(\partial_{s} a \Pi^{+}\right) v^{h} \mid v^{h}\right) & \\
=\left(\operatorname{op}_{h}^{(2)}\left(a \Pi^{+}\right) \partial_{s} v^{h} \mid v^{h}\right)+ & \left(\operatorname{op}_{h}^{(2)}\left(a \Pi^{+}\right) v^{h} \mid \partial_{s} v^{h}\right) \\
& +\left(\operatorname{op}_{h}^{(2)}\left(a \partial_{s} \Pi^{+}\right) v^{h} \mid v^{h}\right) .
\end{aligned}
$$

BULLETIN DE LA SOCiÉtÉ MATHÉMATIQUe DE FRANCE 
Pour estimer la somme $L_{1}^{h}$ des deux premiers termes dans le second membre de (61), on recourt classiquement à l'équation

$$
\frac{h}{i} \partial_{s} v^{h}-\mathrm{op}_{h}(A) v^{h}=f^{h}, \quad \mathrm{op}_{h}(\chi) f^{h}=o(h)
$$

pour tout $\chi \in \mathcal{C}_{0}^{\infty}(U)$. Il vient

$$
\begin{aligned}
L_{1}^{h}=-\frac{i}{h}([ & \left.\left.\operatorname{op}_{h}(A), \mathrm{op}_{h}^{(2)}\left(a \Pi^{+}\right)\right] v^{h} \mid v^{h}\right) \\
& +\frac{i}{h}\left(\left(\operatorname{op}_{h}^{(2)}\left(a \Pi^{+}\right) f^{h} \mid v^{h}\right)-\left(\operatorname{op}_{h}^{(2)}\left(a \Pi^{+}\right) v^{h} \mid f^{h}\right)\right) .
\end{aligned}
$$

En utilisant l'homogénéité en $\left(s, \alpha \zeta_{2}\right)$ de $\Pi^{+}$, on vérifie aisément que $q=a \Pi^{+}$ satisfait aux estimations

$$
\left|\partial_{\sigma, z, \zeta_{1}}^{\beta} \partial_{s, \zeta_{2}}^{\gamma} \tilde{q}_{h}(s, z, \sigma, \zeta)\right| \leq C_{\beta, \gamma}\left(\frac{1}{R \sqrt{h}}\right)^{|\gamma|},
$$

en supposant $R \sqrt{h} \leq 1$, ce qui ne pose pas de problème puisqu'on fait tendre d'abord $h$ vers 0 . Notons que l'on utilise ici que $\alpha \neq 0$ sur $U$, de sorte que $\tilde{\lambda}_{h} \geq C R \sqrt{h}$ sur le support de $\tilde{a}_{h}$. En termes de calcul de Weyl-Hörmander, pour lequel nous référons aux sections 18.4, 18.5, 18.6 de [16], il revient au même de dire que $q_{h}^{\sharp} \in S\left(1, g_{h}\right)$, où $g_{h}$ est la métrique suivante,

$$
g_{h}=\mathrm{d} z^{2}+\frac{\mathrm{d} s^{2}}{R^{2} h}+h^{2}\left(\mathrm{~d} \sigma^{2}+\mathrm{d} \zeta_{1}^{2}\right)+\frac{h \mathrm{~d} \zeta_{2}^{2}}{R^{2}} .
$$

On remarque que

$$
\frac{g_{h}}{g_{h}^{\sigma}} \leq\left(\frac{\sqrt{h}}{R}\right)^{2}
$$

de sorte que le gain du calcul symbolique est majoré par $\sqrt{h} / R$.

Soit alors $\chi \in \mathcal{C}_{0}^{\infty}(U)$ telle que $\chi a=a$. Alors le théorème de calcul symbolique 18.5.4 de [16] assure que

$$
\operatorname{op}_{h}^{(2)}\left(a \Pi^{+}\right)=\operatorname{op}_{h}^{(2)}\left(a \Pi^{+} \chi\right) \in \operatorname{op}_{h}^{(2)}\left(a \Pi^{+}\right) \operatorname{op}_{h}(\chi)+\operatorname{op}_{1}\left(S\left(\left(\frac{\sqrt{h}}{R}\right)^{N}, g_{h}\right)\right)
$$

pour tout entier $N$. Ainsi, d'après le théorème de continuité $L^{2} 18.6 .3$ de [16], on obtient, quand $h$ tend vers 0 ,

$$
\frac{i}{h}\left(\left(\operatorname{op}_{h}^{(2)}\left(a \Pi^{+}\right) f^{h} \mid v^{h}\right)\right)=\frac{i}{h}\left(\left(\operatorname{op}_{h}^{(2)}\left(a \Pi^{+}\right) \operatorname{op}_{h}(\chi) f^{h} \mid v^{h}\right)\right)+o(1)=o(1) .
$$

On a de façon analogue,

$$
\frac{i}{h}\left(\left(\operatorname{op}_{h}^{(2)}\left(a \Pi^{+}\right) v^{h} \mid f^{h}\right)\right)=o(1) .
$$

TOME $130-2002-\mathrm{N}^{\mathrm{O}} 1$ 
Par ailleurs, observons que $A_{h}^{\sharp} \in S\left(\lambda_{h}^{\sharp}, g_{h}\right)$ et que, puisque a est supporté dans un compact fixe, $q_{h}^{\sharp} \in S\left(\left(\lambda_{h}^{\sharp}\right)^{-N}, g_{h}\right)$ pour tout entier $N$. Combiné avec l'identité $A \Pi^{+}=\Pi^{+} A$, le théorème de calcul symbolique assure alors que

$$
\begin{aligned}
{\left[\operatorname{op}_{h}(A), \mathrm{op}_{h}^{(2)}\left(a \Pi^{+}\right)\right]=\left[\mathrm{op}_{1}\left(A_{h}^{\sharp}\right), \mathrm{op}_{1}\left(q_{h}^{\sharp}\right)\right] } & \\
& \in \frac{1}{2 i} \operatorname{op}_{1}\left(\left\{A_{h}^{\sharp}, q_{h}^{\sharp}\right\}-\left\{q_{h}^{\sharp}, A_{h}^{\sharp}\right\}\right)+\mathrm{op}_{1}\left(S\left(\left(\frac{\sqrt{h}}{R}\right)^{2}, g_{h}\right)\right),
\end{aligned}
$$

de sorte que, par le théorème de continuité $L^{2}$, on obtient finalement

$$
L_{1}^{h}=\frac{1}{2}\left(\operatorname{op}_{h}\left(\left\{A, \tilde{a}_{h} \Pi^{+}\right\}-\left\{\tilde{a}_{h} \Pi^{+}, A\right\}\right) v^{h} \mid v^{h}\right)+O\left(\frac{1}{R^{2}}\right) .
$$

De plus, puisque $A=2 \lambda \Pi^{+}-\lambda$ et $\left(\Pi^{+}\right)^{2}=\Pi^{+}$, on vérifie aisément que

$$
\left\{A, \Pi^{+}\right\}-\left\{\Pi^{+}, A\right\}=0 .
$$

Il en résulte que

$$
\left\{A, \tilde{a}_{h} \Pi^{+}\right\}-\left\{\tilde{a}_{h} \Pi^{+}, A\right\}=\left\{A, \tilde{a}_{h}\right\} \Pi^{+}-\Pi^{+}\left\{\tilde{a}_{h}, A\right\}=\tilde{b}_{h},
$$

avec

$$
\begin{aligned}
\begin{aligned}
b(s, z, \sigma, \zeta, \eta)= & -\partial_{\sigma} a\left(J \Pi^{+}+\Pi^{+} J\right) \\
& +\left(\alpha \partial_{z_{2}} a+\zeta_{2} \partial_{\sigma} \alpha \partial_{s} a-\zeta_{2} \partial_{z} \alpha \cdot \partial_{\zeta} a-\partial_{z_{2}} \alpha \eta \partial_{\eta} a\right)\left(L \Pi^{+}+\Pi^{+} L\right),
\end{aligned} \\
\text { et } J=\left(\begin{array}{cc}
1 & 0 \\
0 & -1
\end{array}\right), L=\left(\begin{array}{cc}
0 & 1 \\
1 & 0
\end{array}\right) .
\end{aligned}
$$

En écrivant $\nabla a=\chi \nabla a$ pour $\chi \in \mathcal{C}_{0}^{\infty}\left(U^{\prime}\right)$ valant 1 près du support de $a$, et en appliquant le calcul de Weyl-Hörmander comme précédemment, on peut remplacer $v^{h}$ par $\underline{v}^{h}=\mathrm{op}_{h}(\chi) v^{h}$ dans l'expression ci-dessus de $L_{1}^{h}$. Appliquons alors le lemme 10 au symbole $\tilde{b}_{h}$ ci-dessus. Il vient, quand $h$ tend vers 0 ,

$$
\left|L_{1}^{h}\right| \leq O\left(\frac{1}{R^{2}}\right)+C \int_{-\infty}^{+\infty}\left\{\frac{\varepsilon}{\delta}\left|\rho^{\prime}\left(\frac{s}{\delta}\right)\right|+\rho\left(\frac{s}{\delta}\right)\right\} \mathrm{d} s .
$$

Passant à la limite quand $h$ tend vers 0 , puis quand $R$ tend $+\infty$ et quand $(\varepsilon, \delta)$ tend vers $(0,0)$, on conclut que la contribution de $L_{1}^{h}$ à $(60)$ est nulle.

Il reste à annuler la contribution du troisième terme de (60), c'est-à-dire

$$
L_{2}^{h}=\left(\mathrm{op}_{h}^{(2)}\left(a \partial_{s} \Pi^{+}\right) v^{h} \mid v^{h}\right),
$$

qui représente l'interaction entre les deux modes. On se convainc aisément qu'une application directe du lemme 10 au symbole $\left(a \partial_{s} \Pi^{+}\right)^{r}$ montre seulement que $L_{2}^{h}$ est borné. Pour conclure, nous allons donc utiliser à nouveau l'équation, en partant de l'identité

$$
\partial_{s} \Pi^{+}=\left[A, \frac{A}{2 \lambda^{2}} \partial_{s} \Pi^{+}\right]
$$

BULletin DE LA SOCiÉtÉ MATHÉmATIQUE DE FRANCE 
qui est une conséquence facile de $A=\lambda\left(2 \Pi^{+}-1\right)$ et de $\left(\Pi^{+}\right)^{2}=\Pi^{+}$. Utilisons à nouveau le calcul de Weyl-Hörmander avec la métrique $g_{h}$ précédente. On a $A_{h}^{\sharp} \in S\left(\lambda_{h}^{\sharp}, g_{h}\right)$ et

$$
\left(\frac{a A \partial_{s} \Pi^{+}}{\lambda^{2}}\right)_{h}^{\sharp} \in S\left(\frac{1}{\sqrt{h} R \lambda_{h}^{\sharp}}, g_{h}\right)
$$

d'après l'inégalité $\lambda_{h}^{\#} \geq C \sqrt{h} R$ sur le support de $a_{h}^{\sharp}$. On en déduit

$$
\begin{aligned}
\operatorname{op}_{h}^{(2)}\left(a \partial_{s} \Pi^{+}\right) \in \frac{1}{2} \operatorname{op}_{h}( & A) \operatorname{op}_{h}^{(2)}\left(\frac{a A \partial_{s} \Pi^{+}}{\lambda^{2}}\right) \\
& -\frac{1}{2} \operatorname{op}_{h}^{(2)}\left(\frac{a A \partial_{s} \Pi^{+}}{\lambda^{2}}\right) \operatorname{op}_{h}(A)+\mathrm{op}_{1}\left(S\left(\frac{1}{R^{2}}, g_{h}\right)\right)
\end{aligned}
$$

d'où, en tenant compte de l'équation sur $v^{h}$,

$$
\begin{array}{r}
L_{2}^{h}=O\left(\frac{1}{R^{2}}\right)-\frac{1}{2}\left(\mathrm{op}_{h}^{(2)}\left(a A \partial_{s} \Pi^{+} \lambda^{-2}\right) v^{h}\right. \\
\left.\mid f^{h}\right)+\frac{1}{2}\left(\mathrm{op}_{h}^{(2)}\left(a A \partial_{s} \Pi^{+} \lambda^{-2}\right) f^{h} \mid v^{h}\right) \\
-\frac{h}{2 i}\left(\operatorname{op}_{h}^{(2)}\left(\partial_{s}\left(a A \partial_{s} \Pi^{+} \lambda^{-2}\right)\right) v^{h} \mid v^{h}\right) .
\end{array}
$$

En localisant $v^{h}$ et $f^{h}$ à l'aide d'une troncature $\chi$ comme précédemment, on obtient, quand $h$ tend vers 0 puis $R$ tend vers $+\infty$,

$$
L_{2}^{h}=o(1)+O\left(\frac{1}{R^{2}}\right)-\frac{h}{2 i}\left(\mathrm{op}_{h}^{(2)}\left(\partial_{s}\left(a A \partial_{s} \Pi^{+} \lambda^{-2}\right)\right) \underline{v}^{h} \mid \underline{v}^{h}\right)
$$

où $\underline{v}^{h}=\mathrm{op}_{h}(\chi) v^{h}$. On utilise alors le lemme 10, en tenant compte de

$$
\left|\partial_{\sigma, z}^{\beta} \partial_{s}\left(a A \partial_{s} \Pi^{+} \lambda^{-2}\right)_{h}^{\sim}\right| \leq \frac{C_{\beta}}{\left(s^{2}+h R^{2}\right)^{3 / 2}} .
$$

Il vient finalement

$$
\left|L_{2}^{h}\right| \leq o(1)+O\left(\frac{1}{R^{2}}\right)+C h \int_{-\infty}^{+\infty} \frac{\mathrm{d} s}{\left(s^{2}+h R^{2}\right)^{3 / 2}} \leq o(1)+O\left(\frac{1}{R^{2}}\right),
$$

ce qui achève la démonstration.

\section{Analyse à distance finie}

Dans ce paragraphe, nous allons démontrer les formules de Landau-Zener telles qu'elles sont énoncées dans le théorème 3 pour la partie $\{|\eta|<+\infty\}$ des mesures $\nu^{+}$et $\nu^{-}$.

8.1. Une forme normale à distance finie. - Rappelons que nous travaillons sur le système (53). Commençons par analyser les différents rôles des variables $\sigma$ et $z$ : la variable $z_{1}$ est un simple paramètre, le cas $\alpha=\alpha\left(z_{2}\right)$ se ramène à l'étude du système (10) par un simple changement de variables en $z_{2}$. L'unique difficulté provient de la variable $\sigma$. Nous allons donc montrer que le système (14) admet une forme normale avec $\alpha=\alpha(z)$.

TOME $130-2002-\mathrm{N}^{\mathrm{O}} 1$ 
Proposition 8. - Il existe deux matrices $C_{h}(\sigma, z, \eta)$ et $\tilde{C}_{h}(\sigma, z, \eta)$,

$$
\begin{aligned}
& C_{h}(\sigma, z, \eta)=C_{0}(\sigma, z, \eta)+\sqrt{h} C_{1}(\sigma, z, \eta)+h C_{2}(\sigma, z, \eta), \\
& \tilde{C}_{h}(\sigma, z, \eta)=\tilde{C}_{0}(\sigma, z, \eta)+\sqrt{h} \tilde{C}_{1}(\sigma, z, \eta)+h \tilde{C}_{2}(\sigma, z, \eta),
\end{aligned}
$$

telles que pour tout $a \in \mathcal{C}_{0}^{\infty}\left(\mathbb{R}_{s, \sigma, z, \zeta, \eta}^{7}\right)$,

$$
\left\|\operatorname{op}_{h}^{(2)}(a)\left[\operatorname{op}_{h}^{(2)}\left(\tilde{C}_{h}\right) \operatorname{op}_{h}(Q)-\operatorname{op}_{h}\left(Q_{0}\right) \operatorname{op}_{h}^{(2)}\left(C_{h}\right)\right]\right\|_{\mathcal{L}\left(L^{2}\right)}=O(h \sqrt{h})
$$

avec $C_{0}, \tilde{C}_{0}$ unitaires et

$$
Q_{0}(s, z, \sigma, \zeta)=\left(\begin{array}{cc}
-\sigma+s & \alpha(0, z) \zeta_{2} \\
\alpha(0, z) \zeta_{2} & -\sigma-s
\end{array}\right)
$$

Preuve de la proposition 8. - Soit

$$
J=\left(\begin{array}{rr}
1 & 0 \\
0 & -1
\end{array}\right) \quad \text { et } \quad K=\left(\begin{array}{rr}
0 & 1 \\
-1 & 0
\end{array}\right)
$$

Il est plus commode de travailler avec les matrices $J Q$ et $J Q_{0}$. L'équation (62) devient alors compte tenu de $J^{2}=I$ : pour tout $a \in \mathcal{C}_{0}^{\infty}\left(\mathbb{R}_{s, \sigma, z, \zeta, \eta}^{7}\right)$,

$$
\begin{aligned}
\left\|\operatorname{op}_{h}^{(2)}(a)\left[\operatorname{op}_{h}^{(2)}\left(J \tilde{C}_{h} J\right) \operatorname{op}_{h}(J Q)-\operatorname{op}_{h}\left(J Q_{0}\right) \operatorname{op}_{h}^{(2)}\left(C_{h}\right)\right]\right\|_{\mathcal{L}\left(L^{2}\right)} \\
=O(h \sqrt{h}) .
\end{aligned}
$$

Notons que, puisque $a$ est à support compact dans la variable $\eta$,

$$
\left\|\operatorname{op}_{h}^{(2)}(a) \operatorname{op}_{h}\left(\zeta_{2}\right)\right\|_{\mathcal{L}\left(L^{2}\right)}=\sqrt{h}\left\|\operatorname{op}_{h}^{(2)}(a) \operatorname{op}_{h}^{(2)}(\eta)\right\|_{\mathcal{L}\left(L^{2}\right)}=O(1)
$$

En utilisant le calcul de Weyl, on écrit le premier membre de (63) sous la forme

$$
\left\|\mathrm{op}_{h}^{(2)}(a) \operatorname{op}_{h}^{(2)}\left(A_{0}+\sqrt{h} A_{1}+h A_{2}+O(h \sqrt{h})\right)\right\|_{\mathcal{L}\left(L^{2}\right)} .
$$

On identifie alors $A_{0}, A_{1}$ et $A_{2}$ à 0 . Comme $C_{h}$ et $\tilde{C}_{h}$ ne dépendent pas de $s$, on peut identifier le coefficient de $s$ à 0 , ce qui donne $C_{h}=J \tilde{C}_{h} J$. On obtient les équations suivantes:

$$
\begin{aligned}
{\left[J, C_{0}\right] } & =0, \\
-\sigma\left[J, C_{1}\right]+\alpha(0, z) \eta K C_{0} & =\alpha(\sigma, z) \eta C_{0} K,
\end{aligned}
$$

(66) $-\frac{1}{i} \partial_{\sigma} C_{0}+\frac{1}{2 i}\left(\alpha(0, z) K \partial_{z_{2}} C_{0}-\partial_{z_{2}} \alpha(0, z) \eta K \partial_{\eta} C_{0}\right)$

$$
\begin{gathered}
+\alpha(0, z) \eta K C_{1}-\sigma J C_{2} \\
=-\frac{1}{2 i}\left(\alpha(\sigma, z) \partial_{z_{2}} C_{0} K-\partial_{z_{2}} \alpha(\sigma, z) \eta \partial_{\eta} C_{0} K\right)+\alpha(\sigma, z) \eta C_{1} K-\sigma C_{2} J .
\end{gathered}
$$

BULLETIN DE LA SOCIÉTÉ MATHÉMATIQUE DE FRANCE 
Nous allons maintenant exploiter sucessivement ces trois équations. Notons pour chaque indice $i \in\{0,1,2\}$,

$$
C_{i}=\left(\begin{array}{ll}
a_{i} & c_{i} \\
b_{i} & d_{i}
\end{array}\right) .
$$

L'équation (64) implique que $C_{0}$ est diagonale, $c_{0}(\sigma, z, \eta)=b_{0}(\sigma, z, \eta)=0$.

L'équation (65) ne peut avoir de solutions que si $\left.\alpha(0, z) \eta\left[K, C_{0}\right]\right|_{\sigma=0}=0$, ce qui impose

$$
a_{0}(0, z, \eta)=d_{0}(0, z, \eta)
$$

Sous cette condition, l'équation (65) donne deux équations sur les coefficients antidiagonaux de $C_{1}$, qui sont

$$
\begin{aligned}
& 2 \sigma c_{1}(\sigma, z, \eta)=-\alpha(\sigma, z) \eta a_{0}(\sigma, z, \eta)+\alpha(0, z) \eta d_{0}(\sigma, z, \eta) \\
& 2 \sigma b_{1}(\sigma, z, \eta)=-\alpha(\sigma, z) \eta d_{0}(\sigma, z, \eta)+\alpha(0, z) \eta a_{0}(\sigma, z, \eta)
\end{aligned}
$$

Les coefficient antidiagonaux de l'équation (66) conduisent aux conditions de compatibilité suivantes sur $\sigma=0$ :

$$
\begin{aligned}
d_{1}(0, z, \eta) & =a_{1}(0, z, \eta) \\
\left.\alpha(0, z) \partial_{z_{2}}\left(a_{0}+d_{0}\right)\right|_{\sigma=0} & =\left.\partial_{z_{2}} \alpha(0, z) \eta \partial_{\eta}\left(a_{0}+d_{0}\right)\right|_{\sigma=0} .
\end{aligned}
$$

Modulo ces conditions, les coefficients $b_{2}$ et $c_{2}$ sont déterminés en fonction de $a_{0}, d_{0}, a_{1}$ et $d_{1}$.

En utilisant les équations (69) et (70), les coefficients diagonaux de l'équation (66) conduisent à

$$
\begin{aligned}
& \frac{1}{i} \partial_{\sigma} a_{0}-\frac{\eta^{2}}{2 \sigma}\left(\alpha(0, z)^{2}-\alpha(\sigma, z)^{2}\right) a_{0}=0, \\
& \frac{1}{i} \partial_{\sigma} d_{0}+\frac{\eta^{2}}{2 \sigma}\left(\alpha(0, z)^{2}-\alpha(\sigma, z)^{2}\right) d_{0}=0 .
\end{aligned}
$$

Notons

$$
\beta(\sigma, z)=\frac{1}{\sigma}\left(\alpha(\sigma, z)^{2}-\alpha(0, z)^{2}\right) .
$$

En choisissant $a_{0}(0, z, \eta)=d_{0}(0, z, \eta)=1$ (ce qui assure $(68)$ ), on obtient la solution

$$
a_{0}=\mathrm{e}^{-i \frac{\eta^{2}}{2} \int_{0}^{\sigma} \beta(t, z) \mathrm{d} t}, \quad d_{0}=\mathrm{e}^{i \frac{\eta^{2}}{2} \int_{0}^{\sigma} \beta(t, z) \mathrm{d} t} .
$$

On vérifie alors que (72) est satisfaite. Puis on choisit $d_{1}=a_{1}=a_{2}=d_{2}=0$ : la condition (71) est vérifiée et $b_{1}, c_{1}, b_{2}$ et $c_{2}$ sont alors déterminés. La proposition 8 est démontrée.

TOME $130-2002-\mathrm{N}^{\mathrm{O}} 1$ 
8.2. La formule de Landau-Zener. - Dans ce paragraphe, notre but est d'achever la démonstration du théorème 3. Reprenons les notations du paragraphe 5.2 et utilisons la forme normale du paragraphe 7.1 .

La fonction $v_{0}^{h}=\mathrm{op}_{h}^{(2)}\left(C_{h}\right) v^{h}$ satisfait localement à

$$
\mathrm{op}_{h}\left(Q_{0}\right) v_{0}^{h}=o(h),
$$

et pour $|\eta|<+\infty$, la mesure $\nu_{0}=C_{0}(\sigma, z, \lambda \mu \eta) \underline{\nu} C_{0}(\sigma, z, \lambda \mu \eta)^{*}$ est la mesure à deux échelles de la famille $\left(v_{0}^{h}\right)$ pour l'hypersurface d'équation $\zeta_{2} / \lambda \mu=0$.

Notons $\mu_{0}(z)=\mu(0, z)$. Une transformation canonique $\tilde{\kappa}$ changeant $\zeta_{2} / \mu_{0}$ en $\zeta_{2}$ nous conduit à l'étude de la concentration sur l'hypersurface d'équation $\mu_{0} \zeta_{2} / \lambda \mu=0$ d'une famille de solutions $\left(w^{h}\right)$ du système

$$
\operatorname{op}_{h}\left(\left(\begin{array}{cc}
-\sigma+s & \zeta_{2} \\
\zeta_{2} & -\sigma-s
\end{array}\right)\right) w^{h}=o(h) .
$$

Sa mesure à deux échelles est alors $\nu_{0} \circ \bar{N}(\tilde{\kappa})^{-1}(\eta)$, appelons-la $\rho\left(s, \sigma, z, \zeta_{1}, \eta\right)$. Nous avons

$$
\begin{array}{lll}
\tilde{\rho}=\rho^{+} E_{1}+\rho^{-} E_{2} & \text { au-dessus de } & J^{+, p} \cup J^{-, p}, \\
\tilde{\rho}=\rho^{+} E_{2}+\rho^{-} E_{1} & \text { au-dessus de } & J^{+, f} \cup J^{-, f},
\end{array}
$$

avec, compte tenu de l'expression de $C_{0}$ et de $\underline{\nu}$,

$$
\rho^{ \pm}=\nu^{ \pm} \circ \bar{N}(\tilde{\kappa} \circ \kappa)^{-1}(\eta)
$$

Rappelons que dans les coordonnées $(s, z, \sigma, \zeta)$,

$$
\begin{aligned}
& J^{+, p}=\left\{\zeta_{2}=0, \sigma+s=0, s<0\right\}, \quad J^{-, p}=\left\{\zeta_{2}=0, \sigma-s=0, s<0\right\}, \\
& J^{+, f}=\left\{\zeta_{2}=0, \sigma-s=0, s>0\right\}, \quad J^{-, f}=\left\{\zeta_{2}=0, \sigma+s=0, s>0\right\} .
\end{aligned}
$$

Soient $\rho^{ \pm, p}$ et $\rho^{ \pm, f}$ les traces de $\rho^{ \pm}$sur $S=\left\{\zeta_{2}=\sigma=s=0\right\}$. Notre but est de calculer $\rho^{+, f}$ et $\rho^{-, f}$ en fonction de $\rho^{+, p}$ et $\rho^{-, p}$.

Commençons par établir le lien avec le problème de diffusion de l'appendice. Avec les notations du paragraphe 9, il existe des familles $\alpha_{h}^{ \pm}, \beta_{h}^{ \pm}$telles que la $h$-transformée de Fourier de $w^{h}$ par rapport à $z_{2}$ s'écrive

$$
\begin{aligned}
& \hat{w}^{h}\left(s, z_{1}, \zeta_{2}\right)=\alpha_{h}^{+}\left(z_{1}, \zeta_{2}\right) u_{+}^{+}\left(\frac{s}{\sqrt{h}}, \frac{\zeta_{2}}{\sqrt{h}}\right)+\beta_{h}^{+}\left(z_{1}, \zeta_{2}\right) u_{-}^{+}\left(\frac{s}{\sqrt{h}}, \frac{\zeta_{2}}{\sqrt{h}}\right), \\
& \hat{w}^{h}\left(s, z_{1}, \zeta_{2}\right)=\alpha_{h}^{-}\left(z_{1}, \zeta_{2}\right) u_{+}^{-}\left(\frac{s}{\sqrt{h}}, \frac{\zeta_{2}}{\sqrt{h}}\right)+\beta_{h}^{-}\left(z_{1}, \zeta_{2}\right) u_{-}^{-}\left(\frac{s}{\sqrt{h}}, \frac{\zeta_{2}}{\sqrt{h}}\right)
\end{aligned}
$$

avec

$$
\left(\begin{array}{c}
\alpha_{h}^{+}\left(z_{1}, \zeta_{2}\right) \\
\beta_{h}^{+}\left(z_{1}, \zeta_{2}\right)
\end{array}\right)=S\left(\frac{\zeta_{2}}{\sqrt{h}}\right)\left(\begin{array}{c}
\alpha_{h}^{-}\left(z_{1}, \zeta_{2}\right) \\
\beta_{h}^{-}\left(z_{1}, \zeta_{2}\right)
\end{array}\right) .
$$

BULLETIN DE LA SOCIÉTÉ MATHÉMATIQUe DE FRANCE 
Puisque $w^{h}$ est bornée dans $L_{s}^{\infty}\left(L^{2}\right)$, les fonctions $\alpha_{h}^{ \pm}, \beta_{h}^{ \pm}$sont bornées dans $L^{2}\left(\mathbb{R}^{2}\right)$. Rappelons que notre analyse se situe à distance finie en $\eta=\zeta_{2} / \sqrt{h}$, ce qui autorise l'utilisation des asymptotiques de la proposition 9 . On en déduit

$$
\begin{aligned}
& \begin{aligned}
\hat{w}^{h}\left(s, z_{1}, \zeta_{2}\right)= & \alpha_{h}^{+}\left(z_{1}, \zeta_{2}\right) \mathrm{e}^{i s^{2} / 2 h}\left|\frac{s}{\sqrt{h}}\right|^{i \zeta_{2}^{2} / 2 h}\left(\begin{array}{l}
1 \\
0
\end{array}\right) \\
& +\beta_{h}^{+}\left(z_{1}, \zeta_{2}\right) \mathrm{e}^{-i s^{2} / 2 h}\left|\frac{s}{\sqrt{h}}\right|^{-i \zeta_{2}^{2} / 2 h}\left(\begin{array}{l}
0 \\
1
\end{array}\right)+o(1), \quad s>0, \\
\hat{w}^{h}\left(s, z_{1}, \zeta_{2}\right)= & \alpha_{h}^{-}\left(z_{1}, \zeta_{2}\right) \mathrm{e}^{i s^{2} / 2 h}\left|\frac{s}{\sqrt{h}}\right|^{i \zeta_{2}^{2} / 2 h}\left(\begin{array}{c}
1 \\
0
\end{array}\right) \\
& +\beta_{h}^{-}\left(z_{1}, \zeta_{2}\right) \mathrm{e}^{-i s^{2} / 2 h}\left|\frac{s}{\sqrt{h}}\right|^{-i \zeta_{2}^{2} / 2 h}\left(\begin{array}{l}
0 \\
1
\end{array}\right)+o(1), \quad s<0 .
\end{aligned}
\end{aligned}
$$

En comparant les équations (76) et (77) avec (73) et (74), on constate que

- $\operatorname{pour} s<0, \rho^{+}=\delta(\sigma+s) \otimes \rho_{B^{-}}, \rho^{-}=\delta(\sigma-s) \otimes \rho_{A^{-}}$,

- pour $s>0, \rho^{+}=\delta(\sigma-s) \otimes \rho_{A^{+}}, \rho^{-}=\delta(\sigma+s) \otimes \rho_{B^{+}}$,

où $A^{ \pm}, B^{ \pm}$sont définies par $\hat{A}^{ \pm}=\alpha^{ \pm}, \hat{B}^{ \pm}=\beta^{ \pm}$et $\rho_{F}$ désigne la mesure à deux échelles de $F$ pour l'équation

$$
\mu_{0} \zeta_{2} / \lambda \mu=0
$$

Compte tenu de l'hypothèse $\rho^{-, p} \perp \rho^{+, p}$ et de l'expression de $S(\eta)$ obtenue à la proposition 9 , on obtient la formule

$$
\begin{aligned}
& \rho^{+, f} \\
& \rho^{-, f}
\end{aligned}=\left(\begin{array}{cc}
1-\tilde{T} & \tilde{T} \\
\tilde{T} & 1-\tilde{T}
\end{array}\right)\left(\begin{array}{c}
\rho^{+, p} \\
\rho^{-, p}
\end{array}\right)
$$

avec

$$
\tilde{T}(\eta)=\left|a\left(\frac{\lambda \mu \eta}{\mu_{0}}\right)\right|^{2}=1-\left|b\left(\frac{\lambda \mu \eta}{\mu_{0}}\right)\right|^{2}=\mathrm{e}^{-\pi \lambda^{2} \eta^{2}}
$$

puisque $\mu=\mu_{0}$ sur $S$. En utilisant d'une part les relations (75) entre $\nu$ et $\rho$, d'autre part le fait que $\lambda^{2}|\nabla V(x)|=1$ sur $S$ (formule (46)), on obtient que les traces $\left(\nu_{S}^{+, f}, \nu_{S}^{-, f}\right)$ et $\left(\nu_{S}^{+, p}, \nu_{S}^{-, p}\right)$ de $\nu_{I}$ sur $S$ sont reliées par

$$
\left(\begin{array}{c}
\nu_{S}^{+, f} \\
\nu_{S}^{-}, f
\end{array}\right)=\left(\begin{array}{cc}
1-T & T \\
T & 1-T
\end{array}\right)\left(\begin{array}{c}
\nu_{S}^{+, p} \\
\nu_{S}^{-}, p
\end{array}\right)
$$

avec $T(x, \eta)=\mathrm{e}^{-\pi \eta^{2} /|\nabla V(x)|}$. Cette dernière équation est une formulation équivalente de (13) puisque l'hyperplan $P$ vérifie

$$
P=\left.T I\right|_{S}
$$

Ceci termine la preuve du théorème 3 .

TOME $130-2002-\mathrm{N}^{\mathrm{O}} 1$ 


\section{Appendice : Un problème de diffusion}

Nous nous attachons ici à étudier le problème de la diffusion pour le système $(10)$,

$$
\frac{1}{i} \partial_{s} u=\left(\begin{array}{cc}
s & \eta \\
\eta & -s
\end{array}\right) u
$$

Le but de cet appendice est de donner une démonstration autocontenue du résultat suivant :

Proposition 9. - Il existe $\left(u_{+}^{+}, u_{-}^{+}\right)$et $\left(u_{+}^{-}, u_{-}^{-}\right)$deux bases de solutions de (10) telles que, localement uniformément en $\eta$,

$$
\begin{aligned}
& u_{-}^{-}(s, \eta)=\mathrm{e}^{-i s^{2} / 2}|s|^{-i \eta^{2} / 2}\left(\begin{array}{l}
0 \\
1
\end{array}\right)+o(1), \quad \text { si } s \rightarrow-\infty \\
& u_{+}^{-}(s, \eta)=\mathrm{e}^{i s^{2} / 2}|s|^{i \eta^{2} / 2}\left(\begin{array}{l}
1 \\
0
\end{array}\right)+o(1), \quad \text { si } s \rightarrow-\infty
\end{aligned}
$$

et

$$
\begin{aligned}
& u_{-}^{+}(s, \eta)=\mathrm{e}^{-i s^{2} / 2}|s|^{-i \eta^{2} / 2}\left(\begin{array}{l}
0 \\
1
\end{array}\right)+o(1), \quad \text { si } s \rightarrow+\infty, \\
& u_{+}^{+}(s, \eta)=\mathrm{e}^{i s^{2} / 2}|s|^{i \eta^{2} / 2}\left(\begin{array}{l}
1 \\
0
\end{array}\right)+o(1), \quad \text { si } s \rightarrow+\infty .
\end{aligned}
$$

De plus la matrice unitaire permettant de passer des composantes dans $\left(u_{+}^{-}, u_{-}^{-}\right)$ aux composantes dans la base $\left(u_{+}^{+}, u_{-}^{+}\right)$est

$$
S(\eta)=\left(\begin{array}{rr}
a(\eta) & -\bar{b}(\eta) \\
b(\eta) & a(\eta)
\end{array}\right)
$$

avec

$$
a(\eta)=\mathrm{e}^{-\pi \eta^{2} / 2}, \quad b(\eta)=\frac{2 i}{\sqrt{\pi} \eta} 2^{-i \eta^{2} / 2} \mathrm{e}^{-\pi \eta^{2} / 4} \Gamma\left(1+i \frac{\eta^{2}}{2}\right) \operatorname{sh}\left(\frac{\pi \eta^{2}}{2}\right) .
$$

Démonstration. - Commençons par un changement de coordonnées symplectiques

$$
(s, \sigma) \longmapsto(z, \zeta)=\left(\frac{\sigma-s}{\sqrt{2}}, \frac{\sigma+s}{\sqrt{2}}\right),
$$

quantifié par l'opérateur intégral de Fourier unitaire

$$
v(z)=\frac{1}{\sqrt{\pi \sqrt{2}}} \int \mathrm{e}^{\frac{i}{2}\left(z^{2}+s^{2}-2 \sqrt{2} s z\right)} u(s) \mathrm{d} s .
$$

Après ce changement de fonction inconnue, le système (10) devient

$$
\frac{\sqrt{2}}{i} \partial_{z} v_{1}=\eta v_{2}, \quad \sqrt{2} z v_{2}=\eta v_{1},
$$

BULLETIN DE LA SOCiÉTÉ MATHÉMATIQUe DE FRANCE 
soit encore

$$
z \partial_{z} v_{1}=i \frac{\eta^{2}}{2} v_{1}, \quad v_{2}=\frac{\sqrt{2}}{i \eta} \partial_{z} v_{1} .
$$

Après résolution de ce système, on obtient

$$
u(s, \eta)=\int \mathrm{e}^{-\frac{i}{2}\left(s^{2}+z^{2}-2 \sqrt{2} s z\right)} M(z, \eta)\left(\begin{array}{c}
C(\eta) \\
D(\eta)
\end{array}\right) \mathrm{d} z
$$

où $C(\eta)$ et $D(\eta)$ sont des constantes dépendant du paramètre $\eta$ et

$$
M(z, \eta)=\left(\begin{array}{cc}
|z|^{i \eta^{2} / 2} & \frac{i \eta}{2 \sqrt{2}}|z|^{\frac{i \eta^{2}}{2}} \operatorname{sgn}(z) \\
\frac{\sqrt{2}}{i \eta} \partial_{z}\left(|z|^{i \eta^{2} / 2}\right) & \frac{1}{2} \partial_{z}\left(|z|^{i \eta^{2} / 2} \operatorname{sgn}(z)\right)
\end{array}\right) .
$$

Notre but est alors de déterminer le comportement quand $s$ tend vers $+\infty$ de la matrice

$$
\tilde{M}(s, \eta)=\int M(z, \eta) \exp \left(-\frac{i}{2}\left(s^{2}+z^{2}-2 \sqrt{2} z s\right)\right) \mathrm{d} z
$$

LEMME 11. - Lorsque s tend vers $\pm \infty$

$$
\tilde{M}(s, \eta)=\left(\begin{array}{cc}
\mathrm{e}^{i s^{2} / 2}|s|^{i \eta^{2} / 2} a_{ \pm} & \mathrm{e}^{i s^{2} / 2}|s|^{i \eta^{2} / 2} c_{ \pm} \\
\mathrm{e}^{-i s^{2} / 2}|s|^{-i \eta^{2} / 2} b_{ \pm} & \mathrm{e}^{-i s^{2} / 2}|s|^{-i \eta^{2} / 2} d_{ \pm}
\end{array}\right)+o(1)
$$

avec

$$
\begin{aligned}
& a_{+}(\eta)=a_{-}(\eta)=2^{i \eta^{2} / 4} \sqrt{2 \pi} \mathrm{e}^{-i \pi / 4} \\
& b_{+}(\eta)=-b_{-}(\eta)=\frac{2 i \sqrt{2}}{\eta} 2^{-i \eta^{2} / 4} \Gamma\left(1+i \eta^{2} / 2\right) \operatorname{sh}\left(\pi \eta^{2} / 4\right) \\
& c_{+}(\eta)=-c_{-}(\eta)=i \frac{\eta \sqrt{\pi}}{2} \mathrm{e}^{-i \pi / 4} 2^{i \eta^{2} / 4} \\
& d_{+}(\eta)=d_{-}(\eta)=2^{-i \eta^{2} / 4} \Gamma\left(1+i \eta^{2} / 2\right) \operatorname{ch}\left(\pi \eta^{2} / 4\right)
\end{aligned}
$$

Ce lemme clôt la preuve de la proposition 9 puisque la matrice de scattering $S(\eta)$ est donnée par

$$
S(\eta)=\left(\begin{array}{ll}
a_{+} & c_{+} \\
b_{+} & d_{+}
\end{array}\right)\left(\begin{array}{ll}
a_{-} & c_{-} \\
b_{-} & d_{-}
\end{array}\right)^{-1}
$$

Il nous reste donc à prouver le lemme 11 .

Preuve du lemme 11. - L'outil principal de cette preuve est une méthode de phase stationnaire. Notons

$$
\tilde{M}(s, \eta)=\left(\begin{array}{ll}
a(s, \eta) & c(s, \eta) \\
b(s, \eta) & d(s, \eta)
\end{array}\right)
$$

TOME $130-2002-\mathrm{N}^{\mathrm{O}} 1$ 
avec

$$
\begin{aligned}
& a(s, \eta)=\int \mathrm{e}^{-\frac{i}{2}\left(s^{2}+z^{2}-2 \sqrt{2} s z\right)}|z|^{i \eta^{2} / 2} \mathrm{~d} z, \\
& b(s, \eta)=\frac{\sqrt{2}}{i \eta} \int \mathrm{e}^{-\frac{i}{2}\left(s^{2}+z^{2}-2 \sqrt{2} s z\right)} \frac{\partial}{\partial z}\left(|z|^{i \eta^{2} / 2}\right) \mathrm{d} z, \\
& c(s, \eta)=i \frac{\eta}{2 \sqrt{2}} \int \mathrm{e}^{-\frac{i}{2}\left(s^{2}+z^{2}-2 \sqrt{2} s z\right)}|z|^{i \eta^{2} / 2} \operatorname{sgn}(z) \mathrm{d} z, \\
& d(s, \eta)=\frac{1}{2} \int \mathrm{e}^{-\frac{i}{2}\left(s^{2}+z^{2}-2 \sqrt{2} s z\right)} \frac{\partial}{\partial z}\left(|z|^{i \eta^{2} / 2} \operatorname{sgn}(z)\right) \mathrm{d} z .
\end{aligned}
$$

Le changement de variable $z \mapsto-z$ nous donne

$$
\tilde{M}(-s, \eta)=\left(\begin{array}{cc}
a(s, \eta) & -c(s, \eta) \\
-b(s, \eta) & d(s, \eta)
\end{array}\right)
$$

c'est pourquoi nous nous contenterons d'étudier la limite $s \rightarrow+\infty$.

Coefficient $a$ : après le changement de variable $z=s y, a(s, \eta)$ s'écrit

$$
a(s, \eta)=s^{1+i \eta^{2} / 2} \int \mathrm{e}^{-\frac{i}{2} s^{2}\left(1+y^{2}-2 \sqrt{2} y\right)}|y|^{i \eta^{2} / 2} \mathrm{~d} y .
$$

La phase $\lambda(y)=-\frac{1}{2}\left(1+y^{2}-2 \sqrt{2} y\right)$ a un point critique non-dégénéré en $y=\sqrt{2}$. Comme la fonction $y \mapsto|y|^{i \eta^{2} / 2}$ n'est pas régulière en 0 , nous introduisons une fonction de troncature $\chi \in \mathcal{C}^{\infty}(\mathbb{R}), 0 \leq \chi \leq 1, \chi(y)=0$ pour $|y| \geq \frac{\sqrt{2}}{2}$ et $\chi(y)=1$ pour $|y| \leq \frac{\sqrt{2}}{4}$, qui nous permet de décomposer $a(s, \eta)$ en la somme de deux intégrales :

$$
\begin{aligned}
a(s, \eta)= & s^{1+i \eta^{2} / 2} \int \chi(y) \mathrm{e}^{-\frac{i}{2} s^{2}\left(1+y^{2}-2 \sqrt{2} y\right)}|y|^{i \eta^{2} / 2} \mathrm{~d} y \\
& \quad+s^{1+i \eta^{2} / 2} \int(1-\chi(y)) \mathrm{e}^{-\frac{i}{2} s^{2}\left(1+y^{2}-2 \sqrt{2} y\right)}|y|^{i \eta^{2} / 2} \mathrm{~d} y \\
= & A_{0}+B_{0} .
\end{aligned}
$$

Le théorème de la phase stationnaire nous donne

$$
B_{0}=\sqrt{2 \pi} \mathrm{e}^{-i \pi / 4} 2^{i \eta^{2} / 4} s^{i \eta^{2} / 2} \mathrm{e}^{i s^{2} / 2}+o(1) .
$$

Remarquons que pour $|y| \leq \frac{\sqrt{2}}{2}, y \mapsto y^{2}-2 \sqrt{2} y$ est une fonction strictement décroissante, on peut donc faire dans $A_{0}$ le changement de variables $y=\sqrt{2}-\left(2-2 z / s^{2}\right)^{\frac{1}{2}} ;$ alors

$$
y^{2}-2 \sqrt{2} y+\frac{2 z}{s^{2}}=0 \quad \text { et } \quad s^{2} \mathrm{~d} y=\frac{\mathrm{d} z}{\sqrt{2-2 z / s^{2}}} .
$$

BULLETIN DE LA SOCIÉtÉ MATHÉMATIQUE DE FRANCE 
Il vient

$$
\begin{aligned}
A_{0}=s^{-1+i \eta^{2} / 2} \mathrm{e}^{-i s^{2} / 2} \int \chi\left(\sqrt{2}-\sqrt{2-\frac{2 z}{s^{2}}}\right)\left|\sqrt{2}-\sqrt{2-\frac{2 z}{s^{2}}}\right|^{i \eta^{2} / 2} \\
\times \frac{\mathrm{e}^{i z}}{\sqrt{2-\frac{2 z}{s^{2}}}} \mathrm{~d} z=O\left(\frac{1}{s}\right)
\end{aligned}
$$

en utilisant une méthode d'intégrale oscillante comme dans [2], par exemple. On en déduit $a(s, \eta)=s^{i \eta^{2} / 2} \mathrm{e}^{i s^{2} / 2} a_{+}(\eta)+o(1)$ lorsque $s \rightarrow+\infty$ avec $a_{+}(\eta)=\sqrt{2 \pi} \mathrm{e}^{-i \pi / 4} 2^{i \eta^{2} / 4}$.

Coefficient $b$ : après une intégration par parties, il vient

$$
b(s, \eta)=\frac{\sqrt{2}}{\eta} \int \mathrm{e}^{-\frac{i}{2}\left(s^{2}+z^{2}-2 \sqrt{2} s z\right)}(z-\sqrt{2} s)|z|^{i \eta^{2} / 2} \mathrm{~d} z .
$$

Le changement de variables $z=s y$ nous donne

$$
b(s, \eta)=\frac{\sqrt{2}}{\eta} s^{2+i \eta^{2} / 2} \int \mathrm{e}^{-\frac{i}{2} s^{2}\left(1+y^{2}-2 \sqrt{2} y\right)}(y-\sqrt{2})|y|^{i \eta^{2} / 2} \mathrm{~d} y .
$$

Comme précédemment on va distinguer les deux zones $|y| \leq \frac{\sqrt{2}}{2}$ et $|y| \geq \frac{\sqrt{2}}{2}$ grâce à la même fonction de troncature $\chi$ et on écrit

$$
b(s, \eta)=A_{1}+B_{1} .
$$

Comme la phase est critique en $y_{0}=\sqrt{2}$ et $(y-\sqrt{2})|y|^{i \eta^{2} / 2}$ s'annule en $y_{0}$, on a

$$
B_{1}=O\left(\frac{1}{s}\right)
$$

Dans $A_{1}$ nous effectuons comme précédemment le changement de variable $y=\sqrt{2}-\left(2-2 z / s^{2}\right)^{1 / 2}$ :

$$
\begin{aligned}
A_{1} & =-\frac{\sqrt{2}}{\eta} s^{\frac{i \eta^{2}}{2}} \mathrm{e}^{\frac{-i s^{2}}{2}} \int \sqrt{2-\frac{2 z}{s^{2}}} \cdot\left|\sqrt{2}-\sqrt{2-\frac{2 z}{s^{2}}}\right|^{i \eta^{2} / 2} \chi\left(\sqrt{2}-\sqrt{2-\frac{2 z}{s^{2}}}\right) \\
& =-\frac{\sqrt{2}}{\eta} s^{i \eta^{2} / 2} \mathrm{e}^{-i s^{2} / 2} 2^{-i \eta^{2} / 4} \int \mathrm{e}^{i z}\left|\frac{z}{s^{2}}\right|^{i \eta^{2} / 2} \mathrm{~d} z+o(1) \\
& =-\frac{\sqrt{2}}{\eta} s^{-i \eta^{2} / 2} \mathrm{e}^{-i s^{2} / 2} 2^{-i \eta^{2} / 4} \int \mathrm{e}^{i z}|z|^{i \eta^{2} / 2} \mathrm{~d} z+o(1) .
\end{aligned}
$$

TOME $130-2002-\mathrm{N}^{\mathrm{O}} 1$ 
On obtient alors l'expression de $b_{+}(\eta)$, compte tenu de

$$
\begin{aligned}
\int \mathrm{e}^{i z}|z|^{i \eta^{2} / 2} \mathrm{~d} z & =\int_{0}^{+\infty} \mathrm{e}^{i z} z^{i \eta^{2} / 2} \mathrm{~d} z+\int_{0}^{+\infty} \mathrm{e}^{-i z} z^{i \eta^{2} / 2} \mathrm{~d} z \\
& =i \mathrm{e}^{-\pi \eta^{2} / 4} \Gamma\left(1+i \eta^{2} / 2\right)-i \mathrm{e}^{\pi \eta^{2} / 4} \Gamma\left(1+i \eta^{2} / 2\right) \\
& =-2 i \Gamma\left(1+i \frac{\eta^{2}}{2}\right) \operatorname{sh}\left(\frac{\pi \eta^{2}}{4}\right) .
\end{aligned}
$$

Enfin, les coefficients $c$ et $d$ se traitent respectivement comme les coefficients $a$ et $b$. Ceci achève la démonstration de la proposition 9 .

Remerciements. - Les auteurs remercient S. Alinhac, G. Lebeau ainsi que G. Métivier pour les discussions qu'ils ont eues avec eux au sujet de cet article.

\section{BIBLIOGRAPHIE}

[1] AlinhaC (S.) - Branching of singularities for a class of hyperbolic operators, Indiana Univ. Math. J., t. 27 (1978), no. 6, pp. 1027-1037.

[2] Alinhac (S.) \& GÉRARd (P.) - Opérateurs pseudo-différentiels et théorème de Nash-Moser, InterÉditions-Éditions du CNRS, 1991.

[3] Calderón (A.P.) \& Vaillancourt (R.) - On the boundedness of pseudo-differential operators, J. Math. Soc. Japan, t. 23 (1971), no. 2, pp. 374-378.

[4] Colin de Verdière (Y.) - Sur les singularités de Van Hove génériques, in Analyse globale et physique mathématique (Lyon, 1989), Mém. Soc. Math. France (N.S.), vol. 46, 1991, pp. 99-109.

[5] Colin de Verdière (Y.), Lombardi (M.) \& Pollet (J.) - The microlocal Landau-Zener formula, Ann. Inst. Henri Poincaré, t. 71 (1999), no. 1, pp. 95-127.

[6] Cordoba (A.) \& Fefferman (C.) - Wave packets and Fourier integral operators, Comm. Part. Diff. Eq., t. 3 (1978), pp. 979-1005.

[7] Fermanian Kammerer (C.) - Mesures semi-classiques deux-microlocales, C. R. Acad. Sci. Paris, Série 1 Math., t. 331 (2000), pp. 515-518.

[8] GÉrard (P.) - Mesures semi-classiques et ondes de Bloch, Séminaire E.D.P. de l'École polytechnique, exposé No. XVI, 1991.

[9] _ Microlocal defect measures, Comm. Part. Diff. Eq., t. 16 (1991), pp. $1761-1794$.

[10] GÉrard (P.) \& Leichtnam (E.) - Ergodic Properties of Eigenfunctions for the Dirichlet Problem, Duke Math. J., t. 71 (1993), pp. 559-607.

[11] GÉrard (P.), Markowich (P.A.), Mauser (N.J.) \& Poupaud (F.) - Homogenization Limits and Wigner Transforms, Comm. Pure Appl. Math., t. 50 (1997), no. 4, pp. 323-379.

[12] Hagedorn (G.A.) - Proof of the Landau-Zener formula in an adiabatic limit with small eigenvalue gaps, Commun. Math. Phys., t. 136 (1991), pp. $433-449$.

BULLETIN DE LA SOCIÉTÉ MATHÉMATIQUe DE FRANCE 
[13] Molecular Propagation through Electron Energy Level Crossings, Mem. Amer. Math. Soc., vol. $111 \mathrm{n}^{\circ}$ 536, American Mathematical Society, 1994.

[14] Hagedorn (G.A.) \& Joye (A.) - Landau-Zener transitions through small electronic eigenvalue gaps in the Born-Oppenheimer approximation, Ann. Inst. Henri Poincaré, t. 68 (1998), no. 1, pp. 85-134.

[15] Helgason (S.) - Differential geometry, Lie groups and symetric spaces, Academic Press, 1978.

[16] Hörmander (L.) - The analysis of linear Partial Differential Operators III, Springer-Verlag, 1985.

[17] Hwang (I.L.) - The $L^{2}$ boundedness of pseudo-differential operators, Trans. Amer. Math. Soc., t. 302 (1987), pp. 55-76.

[18] Joye (A.) - Proof of the Landau-Zener formula, Asymptotic Analysis, t. 9 (1994), pp. 209-258.

[19] Kaidi (N.) \& Rouleux (M.) - Forme normale d'un hamiltonien à deux niveaux près d'un point de branchement (limite semi-classique), C. R. Acad. Sci. Paris, Série I Math, t. 317 (1993), no. 4, pp. 359-364.

[20] Landau (L.) - Collected papers of L. Landau, Pergamon Press, 1965.

[21] Lions (P-L.) \& Paul (T.) - Sur les mesures de Wigner, Revista Mat. Iberoamericana, t. 9 (1993), pp. 553-618.

[22] Markowich (P.A.), Mauser (N.J.) \& Poupaud (F.) - A Wigner function approach to semi-classical limits : electrons in a periodic potential, J. Math. Phys., t. 35 (1994), pp. 1066-1094.

[23] Miller (L.) - Propagation d'onde semi-classiques à travers une interface et mesures 2-microlocales, Thèse, École Polytechnique, 1996.

[24] Refraction of high-frequency waves density by sharp interfaces and semiclassical measures at the boundary, J. Math. Pures Appl., t. 79 (2000), pp. $227-269$.

[25] Nier (F.) - A Semi-Classical Picture of Quantum Scattering, Ann. Sci. École Norm. Sup. $4^{e}$ série, t. 29 (1996), pp. 149-183.

[26] Poupaud (F.) \& Ringhofer (C.) - Semi-classical limits in a crystal with exterior potentials and effective mass theorems, Comm. Part. Diff. Eq., t. 21 (1996), no. 11-12, pp. 1897-1918.

[27] Robert (D.) - Autour de l'approximation semi-classique, Birkhaüser, 1983.

[28] Rouleux (M.) - Tunneling effects for $h$ pseudodifferential operators, Feshbach resonances, and the Born-Oppenheimer approximation, in Evolution equations, Feshbach resonances, singular Hodge theory, Math. Top., vol. 16, Wiley-VCH, Berlin, 1999, pp. 131-242.

[29] Zener (C.) - Non-adiabatic crossing of energy levels, Proc. Roy. Soc. Lond., t. 137 (1932), pp. 696-702. 